\title{
Digital Health Interventions for Cardiac Rehabilitation: Systematic Literature Review
}

Shannon Wongvibulsin ${ }^{1}$, PhD; Evagelia E Habeos ${ }^{2}$, MD; Pauline P Huynh ${ }^{1}$, BA; Helen Xun ${ }^{1}$, BS; Rongzi Shan ${ }^{3,4}$, BS; Kori A Porosnicu Rodriguez ${ }^{1}$, BA; Jane Wang ${ }^{1,4}$, MD; Yousuf K Gandapur ${ }^{5}$, MD; Ngozi Osuji ${ }^{3}$, MD, MPH; Lochan M Shah ${ }^{1}$, MD; Erin M Spaulding ${ }^{6}$, PhD, RN, BSN; George Hung ${ }^{1}$, MD; Kellen Knowles ${ }^{7}$, MD; William E Yang ${ }^{1}$, MD; Francoise A Marvel ${ }^{3}$, MD; Eleanor Levin ${ }^{8}$, MD, FACC, FAHA; David J Maron ${ }^{9}$, MD, FACC, FAHA; Neil F Gordon ${ }^{10,11}$, MD, PhD, MPH; Seth S Martin ${ }^{3}$, MD, MHS, FACC, FAHA, FASPC

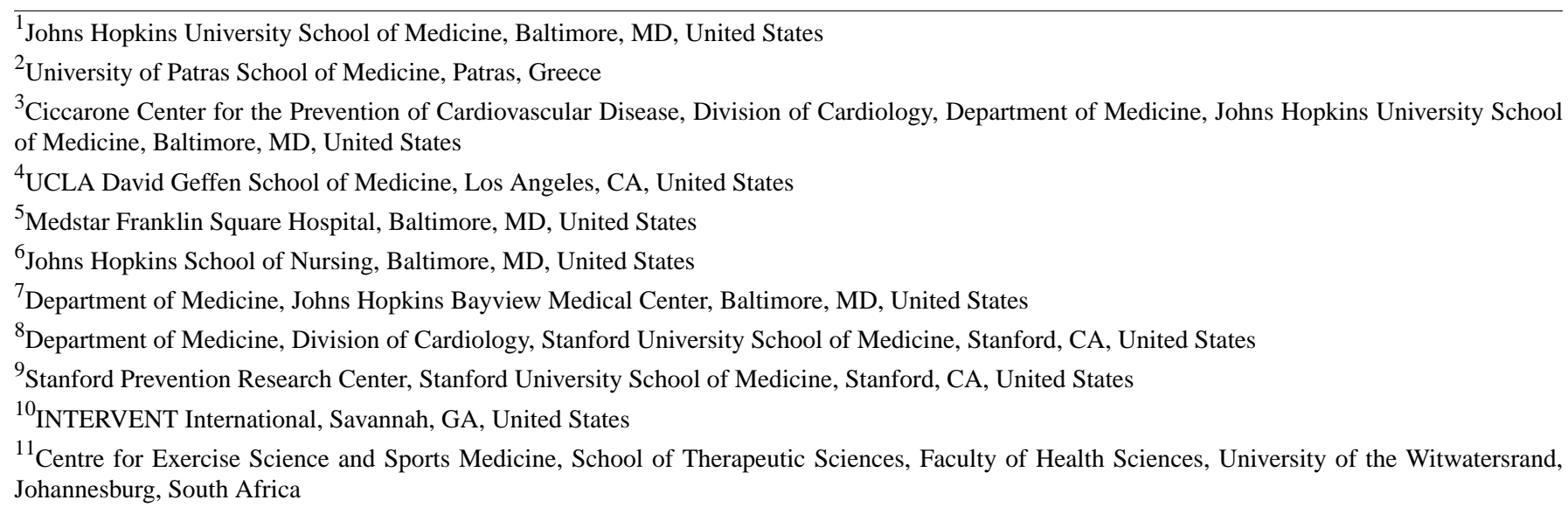

\section{Corresponding Author:}

Seth S Martin, MD, MHS, FACC, FAHA, FASPC

Ciccarone Center for the Prevention of Cardiovascular Disease, Division of Cardiology

Department of Medicine

Johns Hopkins University School of Medicine

$600 \mathrm{~N}$ Wolfe Street

Carnegie 591

Baltimore, MD, 21287

United States

Phone: 14105020469

Email: smart100@jhmi.edu

\section{Abstract}

Background: Cardiovascular disease (CVD) is the leading cause of death worldwide. Despite strong evidence supporting the benefits of cardiac rehabilitation (CR), over $80 \%$ of eligible patients do not participate in CR. Digital health technologies (ie, the delivery of care using the internet, wearable devices, and mobile apps) have the potential to address the challenges associated with traditional facility-based CR programs, but little is known about the comprehensiveness of these interventions to serve as digital approaches to CR. Overall, there is a lack of a systematic evaluation of the current literature on digital interventions for CR.

Objective: The objective of this systematic literature review is to provide an in-depth analysis of the potential of digital health technologies to address the challenges associated with traditional CR. Through this review, we aim to summarize the current literature on digital interventions for CR, identify the key components of CR that have been successfully addressed through digital interventions, and describe the gaps in research that need to be addressed for sustainable and scalable digital CR interventions.

Methods: Our strategy for identifying the primary literature pertaining to CR with digital solutions (defined as technology employed to deliver remote care beyond the use of the telephone) included a consultation with an expert in the field of digital 
CR and searches of the PubMed (MEDLINE), Embase, CINAHL, and Cochrane databases for original studies published from January 1990 to October 2018.

Results: Our search returned 31 eligible studies, of which 22 were randomized controlled trials. The reviewed CR interventions primarily targeted physical activity counseling $(31 / 31,100 \%)$, baseline assessment $(30 / 31,97 \%)$, and exercise training $(27 / 31$, $87 \%)$. The most commonly used modalities were smartphones or mobile devices (20/31, 65\%), web-based portals (18/31, 58\%), and email-SMS (11/31,35\%). Approximately one-third of the studies addressed the CR core components of nutrition counseling, psychological management, and weight management. In contrast, less than a third of the studies addressed other CR core components, including the management of lipids, diabetes, smoking cessation, and blood pressure.

Conclusions: Digital technologies have the potential to increase access and participation in CR by mitigating the challenges associated with traditional, facility-based CR. However, previously evaluated interventions primarily focused on physical activity counseling and exercise training. Thus, further research is required with more comprehensive CR interventions and long-term follow-up to understand the clinical impact of digital interventions.

(J Med Internet Res 2021;23(2):e18773) doi: $\underline{10.2196 / 18773}$

\section{KEYWORDS}

cardiac rehabilitation; telemedicine; digital technologies; mHealth; mobile phone

\section{Introduction}

\section{Cardiac Rehabilitation}

Cardiovascular disease (CVD) is the leading cause of death worldwide, with approximately $80 \%$ of CVD resulting from modifiable risk factors such as physical inactivity, poor dietary habits, elevated low-density lipoprotein-cholesterol and plasma glucose levels, and smoking [1]. Following a cardiac event, cardiac rehabilitation $(\mathrm{CR})$ is an effective modality that enhances recovery, reduces cardiovascular mortality and risk for hospital admissions, and improves the health-related quality of life (QoL) [2]. CR is a multi-faceted, medically supervised program that addresses established core components of guideline-directed therapy, including baseline patient assessments, nutritional counseling, risk factor modification (including management of lipids, blood pressure, weight, diabetes mellitus, and smoking), psychosocial interventions, and physical activity counseling and exercise training [3]. Although there is strong evidence supporting the benefits of CR, less than $20 \%$ of patients who are eligible participate in CR [4]. Challenges related to the low utilization of CR include the lack of referral or facilitation of enrollment, limited health insurance coverage, time and costs associated with participation and travel, and lack of access to a CR facility because of scheduling, transportation, or distance [5].

\section{Digital Technology for CR}

The technology for CR is advancing rapidly and has the potential to address the challenges of traditional facility-based CR programs by delivering care to patients in the convenience of their own homes with real-time, personalized support. As noted in the literature, the terminology describing this technology has not been standardized and includes telemedicine, telehealth, and eHealth [6,7]. In this review, we use the term digital health interventions to encompass technology that enables the delivery of care through means such as the use of the internet, wearable devices, and mobile apps [8,9]. Although there have been encouraging results from the use of digital health interventions for CR (eg, remote electrocardiographic monitoring and mobile or web portal tools), these developments have largely remained in the research settings and have not yet translated into widespread use in clinical practice [3]. Currently, there are gaps in understanding the comprehensiveness of digital CR programs and how successful they are in addressing the core components of CR. To help guide the development of digital CR interventions that have the potential to translate into clinical use, we have focused on the evaluation of technology used in digital interventions for $\mathrm{CR}$ and the comprehensiveness of these programs using the framework outlined in the scientific statement from the American Heart Association (AHA) and the American Association of Cardiovascular and Pulmonary Rehabilitation (AACVPR) for the core components of CR [3]. The specifics regarding the accreditation of CR programs are beyond the scope of this review. With the increasing need for technological advancements to revolutionize the delivery of CR care, this systematic literature review: (1) summarizes the current literature on digital interventions for CR, (2) identifies the key components of CR that have been successfully addressed through digital interventions, and (3) describes the gaps in research that need to be addressed for the sustainable implementation of digital CR interventions in clinical practice.

\section{Methods}

\section{Overview}

We designed a systematic, thematic review to answer key questions regarding the study designs to evaluate $\mathrm{CR}$ interventions, technology used, study size, and comprehensiveness of the investigated interventions. A full list of questions is provided in Textbox 1. Our search terms are detailed in Multimedia Appendix 1. We searched the PubMed (MEDLINE), Embase, CINAHL, and Cochrane databases for studies on digital CR published in English between January 1, 1990, and October 18, 2018. For this review, digital is defined as technology employed to deliver remote care beyond the use of telephone (eg, the delivery of care using the internet, wearable devices, and mobile apps). Telephonic-only studies, which have been addressed in the 2019 scientific statement on home-based CR from the AACVPR, AHA, and American College of Cardiology (ACC) [10], are not within the scope of this review. To determine eligibility for inclusion in this study, titles and 
abstracts were screened for relevance before a full-text review. The inclusion criteria for this review were as follows: (1) original research study using digital or telemedicine approaches for CR and (2) reported results for feasibility, usability, or clinical outcomes. Studies were excluded if they (1) were not full-length publications (ie, abstracts), (2) were methods papers, (3) described only the technology without any inclusion of study participants, or (4) did not include any follow-up time to study outcomes (ie, cross-sectional studies). Given the evolving terminology surrounding digital health technology, we sought external expert inputs to include articles that were not found through our primary search strategy. Papers were included if they reported original research in digital CR. Full details are presented in Figure 1.

Textbox 1. Key questions to evaluate digital cardiac rehabilitation programs.

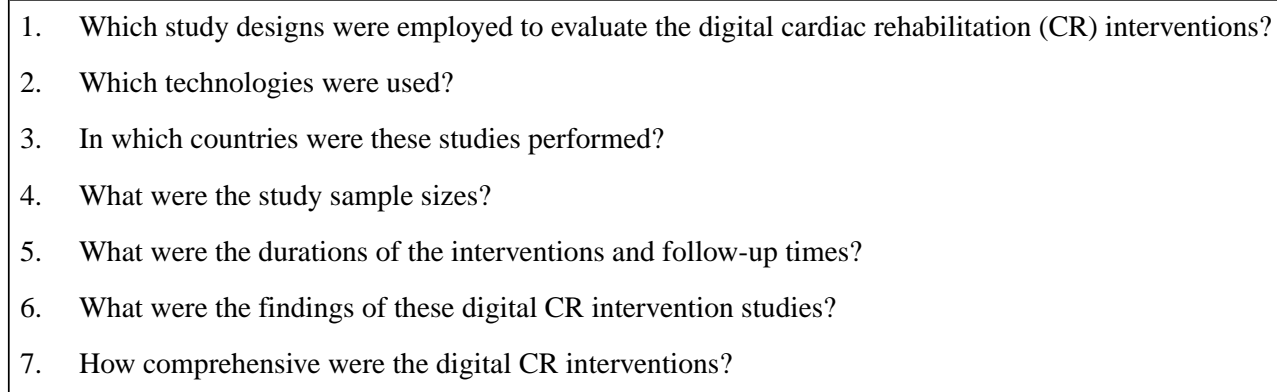

Figure 1. Flowchart for study identification, screening, eligibility, and inclusion. CR: cardiac rehabilitation.

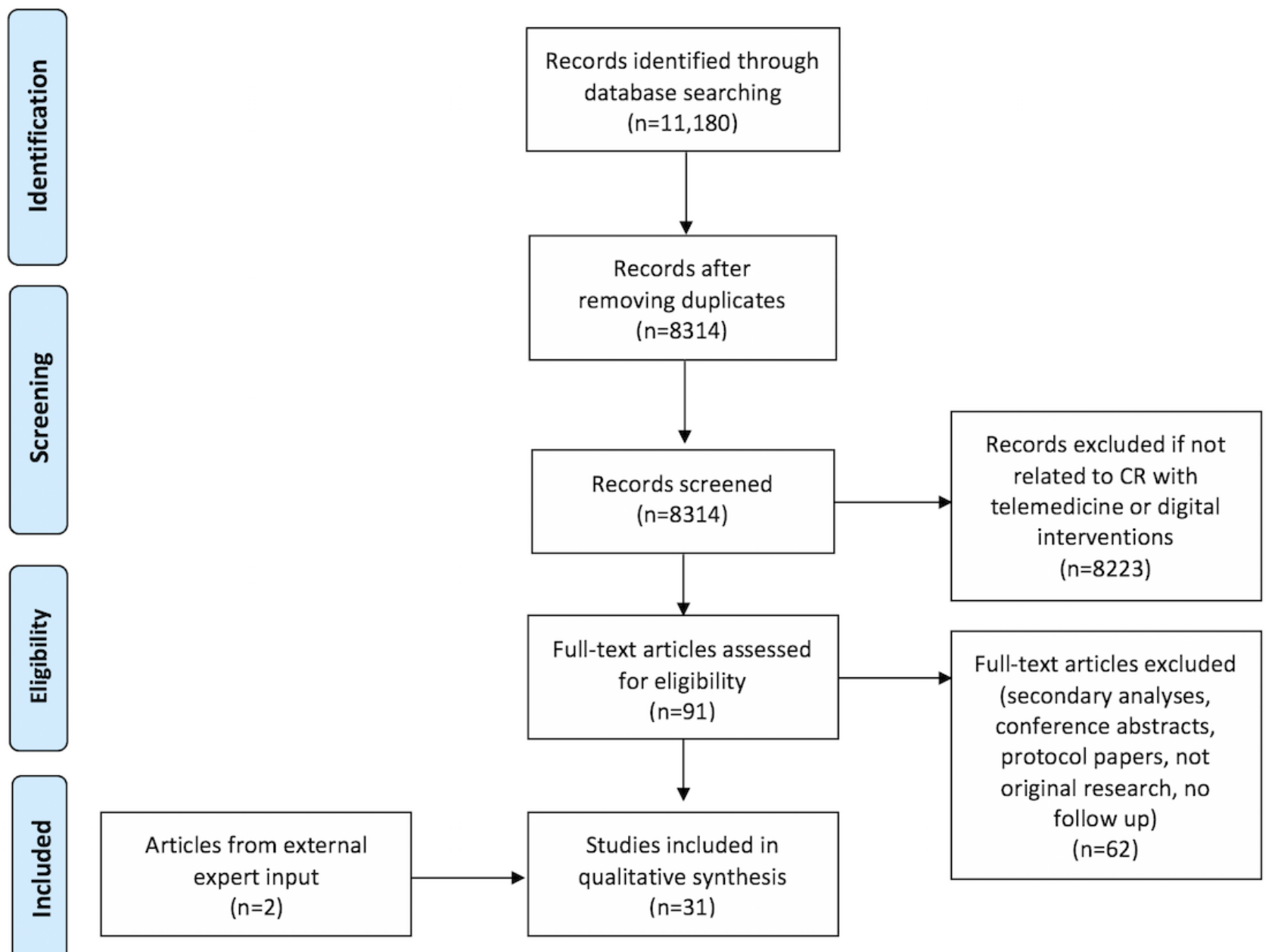

\section{Evaluation of CR Components and Study Quality}

For each study, we recorded the components of CR that were delivered as described in the AHA and AACVPR consensus statement on the core components of $\mathrm{CR}$ and categorized the digital intervention listed in each study as either standalone or adjunctive to conventional CR. Studies were designated as standalone interventions when the program was delivered remotely with the exception of initial in-person session(s) for onboarding or baseline or outcome assessments, as long as the rest of the intervention was remote. The quality of the articles was independently assessed by 2 evaluators using the National Institutes of Health: National Heart, Lung, and Blood Institute (NHI: NHLBI) Study Quality Assessment Tools, which include 
the evaluation of 14 criteria for an overall quality assessment of good, fair, or poor (Multimedia Appendices 2 and 3) [11]. Discrepancies in ratings were resolved by discussions between the evaluators to reach a consensus on the ratings. We followed the guidelines outlined in the Preferred Reporting Items for Systematic Reviews and Meta-Analyses [12].

\section{Results}

\section{Study Characteristics}

In total, 31 studies met the eligibility criteria and were included in this review (Table 1). The study characteristics are summarized in Table 2 and Multimedia Appendix 4. The median sample size was 98 (IQR 52.5-146), the median intervention duration was 3 months (IQR 1.6-4.4 months), and the median follow-up time was 6 months (IQR 3-6 months). The majority of these studies were conducted in Europe $(12 / 31,39 \%)$ and North America $(8 / 31,26 \%)$. A total of 22 studies $(22 / 31,71 \%)$ were randomized controlled trials. Of the 31 studies, 15 (15/31, $48 \%$ ) were standalone digital CR interventions. The study quality was variable, with 23 studies $(23 / 31,74 \%)$ having a good quality, $7(7 / 31,23 \%)$ having a fair quality, and $1(1 / 31$, $3 \%$ ) having a poor quality, according to the criteria established by the NIH: NHLBI Quality Assessment Tools (Multimedia Appendix 5).
As shown in Figure 2, the most commonly targeted CR core components were physical activity counseling (31/31, 100\%), baseline assessment (30/31, 97\%), and exercise training (27/31, $87 \%$ ). Only about one-third of the studies addressed each of the other CR core components of nutrition counseling $(11 / 31,35 \%)$, psychological management $(11 / 31,35 \%)$, and weight management $(10 / 31,32 \%)$. In contrast, less than a third of the studies addressed other CR core components, with only a single study including lipid management $(1 / 31,3 \%), 2$ studies including diabetes management $(2 / 31,6 \%), 7$ studies including tobacco cessation $(7 / 31,23 \%)$, and 8 studies including blood pressure management $(8 / 31,26 \%)$. Smartphones/mobile devices and wearables were employed in $65 \%(20 / 31)$ of the studies, websites or web portals in $58 \%(18 / 31)$, and email-SMS communications in $35 \%(11 / 31)$ of the studies. The interventions were most commonly guided by physical therapists or exercise specialists $(12 / 31,39 \%)$, followed by $\mathrm{CR} /$ research team staff $(11 / 31,35 \%)$, and nurses $(10 / 31,32 \%)$. Four studies $(4 / 31$, $13 \%$ ) described their interventions as fully automated or did not indicate requirement of any specific personnel [13-16]. The most commonly evaluated outcome was exercise capacity or step count $(22 / 31,71 \%)$. Other frequently assessed outcomes included program adherence $(14 / 31,45 \%)$ and QoL (14/31, $45 \%)$. 
Table 1. Characteristics of the included studies.

\begin{tabular}{|c|c|c|c|c|c|c|c|}
\hline Reference & Quality & Country & $\begin{array}{l}\text { Design and } \\
\text { population }\end{array}$ & Intervention & $\begin{array}{l}\mathrm{CR}^{\mathrm{a}} \\
\text { components delivered }^{\mathrm{b}}\end{array}$ & $\begin{array}{l}\text { Personnel and } \\
\text { delivery setting }^{\mathrm{c}}\end{array}$ & Key outcomes \\
\hline $\begin{array}{l}\text { Ades, } 2000 \\
{[17]}\end{array}$ & Fair & $\begin{array}{l}\text { United } \\
\text { States }\end{array}$ & $\begin{array}{l}\text { Nonrandomized } \\
\text { trial of patients } \\
\text { with ACS } \\
\text { within past } 3 \\
\text { months }\end{array}$ & $\begin{array}{l}\text { 3-month home- } \\
\text { based, transtele- } \\
\text { phonically mon- } \\
\text { itored CR, com- } \\
\text { pared with con- } \\
\text { ventional CR }\end{array}$ & $\begin{array}{ll}\text { - } & \mathrm{BA}^{\mathrm{e}} \\
\text { - } & \mathrm{PAC}^{\mathrm{f}} \\
\text { - } & \mathrm{ET}^{\mathrm{g}}\end{array}$ & $\begin{array}{ll}\text { - } & \text { Nurse coordi- } \\
& \text { nator } \\
\text { - } & \text { IPS }^{\mathrm{h}} \\
\text { - } & \text { SAI }^{\mathrm{i}}\end{array}$ & $\begin{array}{ll}\text { - } & \text { Exercise capacity } \\
\text { - } & \text { QoL }^{\mathrm{j}}\end{array}$ \\
\hline $\begin{array}{l}\text { Jenny, } 2001 \\
{[13]}\end{array}$ & Fair & China & $\begin{array}{l}\mathrm{RCT}^{\mathrm{k}} \text { among } \\
\text { cardiac patients } \\
\text { enrolled in } \mathrm{CR}\end{array}$ & $\begin{array}{l}\text { 30-min interac- } \\
\text { tive computer- } \\
\text { based health ed- } \\
\text { ucation pro- } \\
\text { gram, compared } \\
\text { to conventional } \\
\text { health tutorial } \\
\text { sessions }\end{array}$ & $\begin{array}{ll}- & \mathrm{BA} \\
- & \mathrm{PAC}\end{array}$ & $\begin{array}{l}\text { Personnel re- } \\
\text { quired not } \\
\text { specified } \\
\text { Program deliv- } \\
\text { ered through } \\
\text { desktop or } \\
\text { laptop com- } \\
\text { puter }\end{array}$ & $\begin{array}{l}\text { Exercise self-effi- } \\
\text { cacy } \\
\text { - Exercise knowl- } \\
\text { edge }\end{array}$ \\
\hline $\begin{array}{l}\text { Gordon, } \\
2002[18]\end{array}$ & Good & $\begin{array}{l}\text { United } \\
\text { States }\end{array}$ & $\begin{array}{l}\text { RCT among } \\
\text { CAD }^{1} \text { patients }\end{array}$ & $\begin{array}{l}\text { 12-week physi- } \\
\text { cian-supervised, } \\
\text { nurse-case- } \\
\text { managed cardio- } \\
\text { vascular risk re- } \\
\text { duction pro- } \\
\text { gram and a } \\
\text { community- } \\
\text { based cardiovas- } \\
\text { cular risk reduc- } \\
\text { tion program } \\
\text { (including } \\
\text { counseling via } \\
\text { the telephone } \\
\text { and internet) to } \\
\text { patients with } \\
\text { low-to-moder- } \\
\text { ate-risk CAD as } \\
\text { compared to } \\
\text { contemporary } \\
\text { phase II CR } \\
\text { program }\end{array}$ & $\begin{array}{ll}\text { - } & \mathrm{BA} \\
\text { - } & \mathrm{NC}^{\mathrm{m}} \\
\text { - } & \mathrm{WM}^{\mathrm{n}} \\
\text { - } & \mathrm{LM}^{\mathrm{o}} \\
\text { - } & \mathrm{TC}^{\mathrm{p}} \\
\text { - } & \mathrm{PAC} \\
\text { - } & \mathrm{ET} \\
\text { - } & \mathrm{PM}^{\mathrm{q}}\end{array}$ & $\begin{array}{l}\text { Physician-su- } \\
\text { pervised pro- } \\
\text { gram: physi- } \\
\text { cian, nurse- } \\
\text { case manager } \\
\text { - IPS } \\
\text { - Community- } \\
\text { based pro- } \\
\text { gram: exer- } \\
\text { cise physiolo- } \\
\text { gists, non- } \\
\text { physician } \\
\text { - Health } \\
\text { care pro- } \\
\text { fession- } \\
\text { als } \\
\text { Physi- } \\
\text { cians } \\
\text { IPS }\end{array}$ & $\begin{array}{ll}\text { - } & \text { Maximal oxygen } \\
\text { - } & \text { BPtake } \\
\text { - } & \text { Weight } \\
\text { - } & \text { Lipid profile } \\
\text { - } & \text { Medication use }\end{array}$ \\
\hline $\begin{array}{l}\text { Southard, } \\
2003 \text { [19] }\end{array}$ & Good & $\begin{array}{l}\text { United } \\
\text { States }\end{array}$ & $\begin{array}{l}\text { RCT among } \\
\mathrm{CVD}^{\mathrm{r}} \text { patients }\end{array}$ & $\begin{array}{l}\text { 6-month inter- } \\
\text { net-based pro- } \\
\text { gram containing } \\
\text { risk factor man- } \\
\text { agement sup- } \\
\text { port, education, } \\
\text { and monitoring } \\
\text { services to pa- } \\
\text { tients with } \\
\text { CVD, as com- } \\
\text { pared to usual } \\
\text { care }\end{array}$ & $\begin{array}{ll}\text { - } & \mathrm{BA} \\
\text { - } & \mathrm{NC} \\
\text { - } & \mathrm{BPM} \\
\text { - } & \mathrm{PAC} \\
\text { - } & \mathrm{ET}\end{array}$ & $\begin{array}{ll}\text { - } & \text { Case manag- } \\
\text { er, dietician } \\
\text { - } & \text { IPS } \\
\text { - } & \text { SAI }\end{array}$ & $\begin{array}{ll}\text { - } & \text { Satisfaction } \\
\text { - } & \text { Participation } \\
\text { - } & \text { Cost-effectiveness } \\
\text { - } & \text { Weight } \\
\text { - } & \text { BP } \\
\text { - } & \text { Lipid profile } \\
\text { - } & \text { Depression } \\
\text { - } & \text { Exercise capacity } \\
\text { - } & \text { Dietary habits }\end{array}$ \\
\hline $\begin{array}{l}\text { Barnason, } \\
2009 \text { [14] }\end{array}$ & Good & $\begin{array}{l}\text { United } \\
\text { States }\end{array}$ & $\begin{array}{l}\text { RCT among } \\
\text { CABG }^{\mathrm{s}} \text { patients }\end{array}$ & $\begin{array}{l}\text { 6-week symp- } \\
\text { tom manage- } \\
\text { ment telehealth } \\
\text { intervention } \\
\text { comprised of } \\
\text { questionnaires, } \\
\text { accelerometer, } \\
\text { activity diary } \\
\text { compared to } \\
\text { standard of care }\end{array}$ & $\begin{array}{ll}- & \text { BA } \\
- & \text { PAC } \\
- & \text { ET }\end{array}$ & None specified & Exercise capacity \\
\hline
\end{tabular}




\begin{tabular}{|c|c|c|c|c|c|c|c|}
\hline Reference & Quality & Country & $\begin{array}{l}\text { Design and } \\
\text { population }\end{array}$ & Intervention & $\begin{array}{l}\mathrm{CR}^{\mathrm{a}} \\
\text { components delivered }^{\mathrm{b}}\end{array}$ & $\begin{array}{l}\text { Personnel and } \\
\text { delivery setting }^{c}\end{array}$ & Key outcomes \\
\hline $\begin{array}{l}\text { Scalvini, } \\
2009 \text { [20] }\end{array}$ & Good & Italy & $\begin{array}{l}\text { Pilot study of } \\
\text { patients with } \\
\text { postop CABG } \\
\text { or valve surgery }\end{array}$ & $\begin{array}{l}\text { 1-month home- } \\
\text { based CR with } \\
\text { remotely trans- } \\
\text { mitted ECGs }\end{array}$ & $\begin{array}{ll}- & \text { BA } \\
- & \text { PAC } \\
- & \text { ET }\end{array}$ & $\begin{array}{ll}\text { - } & \text { Nurse-tutor, } \\
\text { physiothera- } \\
\text { pist } \\
\text { - } & \text { GS }^{\mathrm{u}} \\
\text { - } & \text { IPS } \\
\text { - } & \text { SAI }\end{array}$ & Step count \\
\hline $\begin{array}{l}\text { Piotrowicz, } \\
2010 \text { [21] }\end{array}$ & Fair & Poland & $\begin{array}{l}\text { RCT among pa- } \\
\text { tients with } \mathrm{HF}^{\mathrm{v}}\end{array}$ & $\begin{array}{l}\text { 8-week home- } \\
\text { based telemoni- } \\
\text { tored CR, com- } \\
\text { pared with con- } \\
\text { ventional CR }\end{array}$ & $\begin{array}{ll}\text { - } & \mathrm{BA} \\
\text { - } & \mathrm{WM} \\
\text { - } & \mathrm{BPM}^{\mathrm{W}} \\
\text { - } & \mathrm{PAC} \\
\text { - } & \mathrm{ET} \\
\text { - } & \mathrm{PM}\end{array}$ & 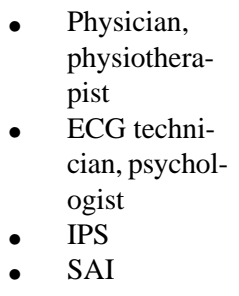 & $\begin{array}{l}\text { - Intervention adher- } \\
\text { ence } \\
\text { - } \quad \text { Exercise capacity }\end{array}$ \\
\hline $\begin{array}{l}\text { Reid, } 2011 \\
\text { [22] }\end{array}$ & Good & Canada & $\begin{array}{l}\text { RCT among } \\
\text { CHD patients } \\
\text { not participat- } \\
\text { ing in CR }\end{array}$ & $\begin{array}{l}\text { 6-month web- } \\
\text { based tailored } \\
\text { exercise inter- } \\
\text { vention with } \\
\text { email coaching, } \\
\text { compared to } \\
\text { standard of care }\end{array}$ & $\begin{array}{ll}- & \text { BA } \\
- & \text { PAC } \\
- & \text { ET }\end{array}$ & $\begin{array}{ll}\text { - } & \text { Exercise spe- } \\
\text { cialist } \\
\text { - } & \text { IPS } \\
\text { - } & \text { SAI }\end{array}$ & Exercise capacity \\
\hline $\begin{array}{l}\text { Clark, } 2013 \\
{[23]}\end{array}$ & Fair & Australia & $\begin{array}{l}\text { Pilot study of } \\
\text { patients with } \\
\text { post-MI }{ }^{\mathrm{x}} \text { or an- } \\
\text { gioplasty }\end{array}$ & $\begin{array}{l}\text { 7-week web- } \\
\text { based CR inter- } \\
\text { vention with ed- } \\
\text { ucational materi- } \\
\text { als, workbooks, } \\
\text { and discussion } \\
\text { forums, glucose } \\
\text { and } \mathrm{BP}^{\mathrm{y}} \text { moni- } \\
\text { toring, and pe- } \\
\text { dometer }\end{array}$ & $\begin{array}{ll}\text { - } & \mathrm{BA} \\
\text { - } & \mathrm{NC} \\
\text { - } & \mathrm{WM} \\
\text { - } & \mathrm{BPM} \\
\text { - } & \mathrm{DM}^{\mathrm{Z}} \\
\text { - } & \mathrm{TC} \\
\text { - } & \mathrm{PAC} \\
\text { - } & \mathrm{PM}\end{array}$ & $\begin{array}{l}\text { - General practi- } \\
\text { tioner, nurse, } \\
\text { allied health } \\
\text { professional, } \\
\text { case manager } \\
\text { - } \text { IPS } \\
\text { - SAI }\end{array}$ & Engagement \\
\hline $\begin{array}{l}\text { Brough, } \\
2014 \text { [24] }\end{array}$ & Fair & $\begin{array}{l}\text { United } \\
\text { Kingdom }\end{array}$ & $\begin{array}{l}\text { Pilot study of } \\
\text { patients with } \\
\mathrm{CHD}^{\text {aa }} \text { referred } \\
\text { for } \mathrm{CR}\end{array}$ & $\begin{array}{l}\text { 8-week web- } \\
\text { based CR com- } \\
\text { prised of web- } \\
\text { based coaching } \\
\text { and exercise e- } \\
\text { diary }\end{array}$ & $\begin{array}{ll}- & \mathrm{BA} \\
- & \mathrm{NC} \\
- & \mathrm{WM} \\
- & \mathrm{TC} \\
- & \mathrm{PAC} \\
- & \mathrm{ET} \\
- & \mathrm{PM}\end{array}$ & $\begin{array}{ll}\text { - } & \text { CR specialist } \\
\text { - } & \text { IPS } \\
\text { - } & \text { SAI }\end{array}$ & $\begin{array}{ll}\text { - } & \text { Exercise capacity } \\
\text { - } & \text { Nutrition } \\
\text { Psychosocial well- } \\
\text { being }\end{array}$ \\
\hline $\begin{array}{l}\text { Devi, } 2014 \\
{[25]}\end{array}$ & Good & England & $\begin{array}{l}\text { RCT among pa- } \\
\text { tients with CHD }\end{array}$ & $\begin{array}{l}\text { 6-week web- } \\
\text { based CR com- } \\
\text { prised of exer- } \\
\text { cise diary and } \\
\text { web-based } \\
\text { coaching, com- } \\
\text { pared to stan- } \\
\text { dard of care }\end{array}$ & $\begin{array}{ll}\text { - } & \mathrm{BA} \\
- & \mathrm{NC} \\
- & \mathrm{WM} \\
- & \mathrm{TC} \\
- & \mathrm{PAC} \\
- & \mathrm{ET} \\
- & \mathrm{PM}\end{array}$ & $\begin{array}{ll}\text { - } & \text { Researcher } \\
\text { - } & \text { IPS } \\
\text { - } & \text { SAI }\end{array}$ & $\begin{array}{ll}\text { - } & \text { Step count } \\
\text { - } & \text { Exercise capacity } \\
\text { - } & \text { Weight } \\
\text { - } & \text { BP } \\
\text { - } & \text { Body fat percent- } \\
& \text { age } \\
\text { - } & \text { QoL measures } \\
\text { - } & \text { Self-efficacy } \\
\text { - } & \text { Anxiety or depres- } \\
\text { - } & \text { Sion } \\
\text { Dietary habits }\end{array}$ \\
\hline $\begin{array}{l}\text { Forman, } \\
2014 \text { [26] }\end{array}$ & Good & $\begin{array}{l}\text { United } \\
\text { States }\end{array}$ & $\begin{array}{l}\text { Pilot study of } \\
\text { patients en- } \\
\text { rolled in CR }\end{array}$ & & $\begin{array}{ll}- & \text { PAC } \\
- & \text { ET } \\
- & \text { PM }\end{array}$ & $\begin{array}{l}\text { Nurse manager, } \\
\text { exercise physiolo- } \\
\text { gist, nutritionist }\end{array}$ & Engagement \\
\hline
\end{tabular}




\begin{tabular}{|c|c|c|c|c|c|c|c|}
\hline Reference & Quality & Country & $\begin{array}{l}\text { Design and } \\
\text { population }\end{array}$ & Intervention & $\begin{array}{l}\mathrm{CR}^{\mathrm{a}} \\
\text { components delivered }\end{array}$ & $\begin{array}{l}\text { Personnel and } \\
\text { delivery setting }\end{array}$ & Key outcomes \\
\hline & & & & $\begin{array}{l}\text { 30-day task- } \\
\text { based smart- } \\
\text { phone CR inter- } \\
\text { vention com- } \\
\text { prised of medi- } \\
\text { cation and } \\
\text { walking re- } \\
\text { minders, sur- } \\
\text { veys, and educa- } \\
\text { tional tools, } \\
\text { with web-based } \\
\text { monitoring }\end{array}$ & & & \\
\hline $\begin{array}{l}\text { Kraal, } 2014 \\
{[27]}\end{array}$ & Good & $\begin{array}{l}\text { Nether- } \\
\text { lands }\end{array}$ & $\begin{array}{l}\text { RCT among } \\
\text { low- to moder- } \\
\text { ate-risk CR pa- } \\
\text { tients }\end{array}$ & $\begin{array}{l}\text { 12-week home- } \\
\text { based CR with } \\
\text { telemonitored } \\
\text { coaching inter- } \\
\text { ventions com- } \\
\text { pared to stan- } \\
\text { dard of care }\end{array}$ & $\begin{array}{ll}\text { - } & \text { BA } \\
\text { - } & \text { PAC } \\
\text { - } & \text { ET }\end{array}$ & $\begin{array}{l}\text { - } \\
\text { physical thera- } \\
\text { - } \\
\text { IPS }\end{array}$ & $\begin{array}{l}\text { - } \quad \text { Exercise capacity } \\
\text { - } \quad \text { QoL }\end{array}$ \\
\hline $\begin{array}{l}\text { Piotrowicz, } \\
2014 \text { [28] }\end{array}$ & Poor & Poland & $\begin{array}{l}\text { Nonrandomized } \\
\text { trial of CVD pa- } \\
\text { tients referred } \\
\text { for outpatient } \\
\text { phase II CR }\end{array}$ & $\begin{array}{l}\text { 4-week home- } \\
\text { based CR with } \\
\text { remote ECG } \\
\text { monitoring with } \\
\text { mobile phone } \\
\text { transmission }\end{array}$ & $\begin{array}{ll}\text { - } & \text { BA } \\
\text { - } & \text { PAC } \\
\text { - } & \text { ET }\end{array}$ & $\begin{array}{ll}- & \text { Nurse } \\
\text { - } & \text { IPS } \\
\text { - } & \text { SAI }\end{array}$ & $\begin{array}{ll}\text { - } & \text { Intervention adher- } \\
\text { ence } \\
\text { - } & \text { Satisfaction } \\
\text { - } & \text { Exercise capacity }\end{array}$ \\
\hline $\begin{array}{l}\text { Varnfield, } \\
2014 \text { [29] }\end{array}$ & Fair & Australia & $\begin{array}{l}\text { RCT among } \\
\text { post-MI pa- } \\
\text { tients referred } \\
\text { to CR }\end{array}$ & $\begin{array}{l}\text { 6-week home- } \\
\text { based CR using } \\
\text { smartphone in- } \\
\text { terventions (edu- } \\
\text { cational materi- } \\
\text { als, exercise } \\
\text { monitoring, } \\
\text { weekly coach- } \\
\text { ing), compared } \\
\text { with convention- } \\
\text { al CR }\end{array}$ & $\begin{array}{ll}\text { - } & \mathrm{BA} \\
\text { - } & \mathrm{NC} \\
\text { - } & \mathrm{WM} \\
\text { - } & \mathrm{BPM} \\
\text { - } & \mathrm{TC} \\
\text { - } & \mathrm{PAC} \\
\text { - } & \mathrm{EM}\end{array}$ & $\begin{array}{ll}\text { - } & \begin{array}{l}\text { Mentor } \\
\text { (health coach) }\end{array} \\
\text { - } & \text { IPS } \\
\text { - } & \text { SAI }\end{array}$ & $\begin{array}{ll}\text { - } & \text { Intervention adher- } \\
& \text { ence } \\
\text { - } & \text { QoL } \\
\text { - } & \text { Exercise capacity } \\
\text { - } & \text { Weight }\end{array}$ \\
\hline $\begin{array}{l}\text { Whittaker, } \\
2014 \text { [30] }\end{array}$ & Fair & Australia & $\begin{array}{l}\text { RCT among pa- } \\
\text { tients at post- } \\
\text { MI }\end{array}$ & $\begin{array}{l}\text { 6-week home } \\
\text { telehealth-based } \\
\text { CR comprising } \\
\text { mobile phone, } \\
\text { Wellness Diary } \\
\text { and web portal } \\
\text { with tele-coach- } \\
\text { ing, as com- } \\
\text { pared to hospi- } \\
\text { tal-based CR }\end{array}$ & $\begin{array}{ll}\text { - } & \text { BA } \\
\text { - } & \text { PAC } \\
\text { - } & \text { ET }\end{array}$ & $\begin{array}{ll}\text { - } & \text { Health coach } \\
\text { - } & \text { IPS } \\
\text { - } & \text { SAI }\end{array}$ & $\begin{array}{ll}\text { - } & \text { Health outcomes } \\
\text { - } & \text { Efficacy } \\
\text { - } & \text { Participation } \\
& \text { Cost-effectiveness }\end{array}$ \\
\hline $\begin{array}{l}\text { Pfaeffli } \\
\text { Dale, } 2015 \\
{[16]}\end{array}$ & Good & $\begin{array}{l}\text { New } \\
\text { Zealand }\end{array}$ & $\begin{array}{l}\text { Qualitative sur- } \\
\text { vey of patients } \\
\text { with CHD }\end{array}$ & $\begin{array}{l}\text { 24-week mobile } \\
\text { health program } \\
\text { comprising text } \\
\text { messaging and } \\
\text { web-based } \\
\text { coaching plus } \\
\text { center-based } \\
\text { CR, compared } \\
\text { to center-based } \\
\text { CR alone }\end{array}$ & $\begin{array}{ll}\text { - } & \mathrm{BA} \\
- & \mathrm{NC} \\
\text { - } & \mathrm{TC} \\
\text { - } & \mathrm{PAC} \\
\text { - } & \mathrm{PM}\end{array}$ & 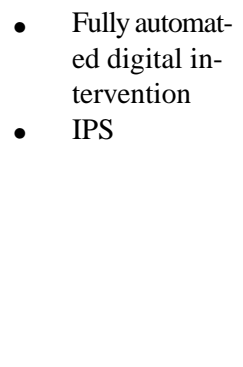 & $\begin{array}{ll}\text { - } & \text { Lifestyle modifica- } \\
\text { tion } \\
\text { - } & \text { QoL } \\
\text { - } & \text { Intervention adher- } \\
\text { ence }\end{array}$ \\
\hline $\begin{array}{l}\text { Frederix, } \\
2015 \text { [31] }\end{array}$ & Good & Belgium & $\begin{array}{l}\text { RCT among } \\
\text { CAD patients } \\
\text { who completed } \\
\text { phase II CR }\end{array}$ & $\begin{array}{l}18 \text {-week tele- } \\
\text { monitored exer- } \\
\text { cise program, } \\
\text { compared with } \\
\text { standard of care }\end{array}$ & $\begin{array}{ll}\text { - } & \text { BA } \\
\text { - } & \text { PAC } \\
\text { - } & \text { ET }\end{array}$ & $\begin{array}{ll}\text { - } & \text { Rehabilitation } \\
\text { center staff } \\
\text { - } & \text { IPS }\end{array}$ & $\begin{array}{ll}\text { - } & \text { Exercise capacity } \\
\text { - } & \text { Weight } \\
\text { - } & \text { Lipid profile } \\
\text { - } & \text { Glycemic control } \\
& \text { Rehospitalizations }\end{array}$ \\
\hline
\end{tabular}




\begin{tabular}{|c|c|c|c|c|c|c|c|}
\hline Reference & Quality & Country & $\begin{array}{l}\text { Design and } \\
\text { population }\end{array}$ & Intervention & $\begin{array}{l}\mathrm{CR}^{\mathrm{a}} \\
\text { components delivered }^{\mathrm{b}}\end{array}$ & $\begin{array}{l}\text { Personnel and } \\
\text { delivery setting }^{\mathrm{c}}\end{array}$ & Key outcomes \\
\hline $\begin{array}{l}\text { Lear, } 2015 \\
{[32]}\end{array}$ & Good & Canada & $\begin{array}{l}\text { RCT among pa- } \\
\text { tients with ACS } \\
\text { or postrevascu- } \\
\text { larization }\end{array}$ & $\begin{array}{l}\text { 4-month web- } \\
\text { based CR com- } \\
\text { prising educa- } \\
\text { tion, coaching, } \\
\text { and physiologic } \\
\text { data monitor- } \\
\text { ing, compared } \\
\text { with standard of } \\
\text { care }\end{array}$ & $\begin{array}{ll}\text { - } & \text { BA } \\
\text { - } & \text { NC } \\
\text { - } & \text { WM } \\
\text { - } & \text { BPM } \\
\text { - } & \text { DM } \\
\text { - } & \text { PAC } \\
\text { - } & \text { ET }\end{array}$ & $\begin{array}{ll}\text { - } & \text { Program } \\
\text { nurse, case } \\
\text { manager, exer- } \\
\text { cise special- } \\
\text { ist, dietician } \\
\text { - } \quad \text { GS } \\
\text { - } \quad \text { IPS } \\
\text { SAI }\end{array}$ & $\begin{array}{ll}\text { - } & \text { Exercise capacity } \\
\text { - } & \text { Lipid profile } \\
\text { - } & \text { Dietary outcomes }\end{array}$ \\
\hline $\begin{array}{l}\text { Maddison, } \\
2015 \text { [15] }\end{array}$ & Good & $\begin{array}{l}\text { New } \\
\text { Zealand }\end{array}$ & $\begin{array}{l}\text { RCT among pa- } \\
\text { tients with } \\
\text { IHD }^{\text {ab }}\end{array}$ & $\begin{array}{l}\text { 24-week smart- } \\
\text { phone-based in- } \\
\text { tervention } \\
\text { (website, educa- } \\
\text { tional videos, } \\
\text { text messaging) } \\
\text { plus standard of } \\
\text { care, compared } \\
\text { with standard of } \\
\text { care alone }\end{array}$ & $\begin{array}{ll}- & \mathrm{BA} \\
- & \mathrm{NC} \\
- & \mathrm{PAC} \\
- & \mathrm{ET} \\
- & \mathrm{PM}\end{array}$ & Not specified & $\begin{array}{ll}\text { - } & \text { Exercise capacity } \\
\text { - } & \text { QoL } \\
\text { - } & \text { Cost-effectiveness }\end{array}$ \\
\hline $\begin{array}{l}\text { Smolis-Bak, } \\
2015 \text { [33] }\end{array}$ & Good & Poland & $\begin{array}{l}\text { Prospective ran- } \\
\text { domized study } \\
\text { among patients } \\
\text { with HF and } \\
\text { implanted CRT- } \\
\text { D }^{\text {ac }}\end{array}$ & $\begin{array}{l}\text { 8-week telemon- } \\
\text { itored home- } \\
\text { based CR, com- } \\
\text { pared to no } \\
\text { training pro- } \\
\text { gram after dis- } \\
\text { charge }\end{array}$ & $\begin{array}{ll}- & \text { BA } \\
- & \text { PAC } \\
- & \text { ET }\end{array}$ & $\begin{array}{l}\text { - CR center } \\
\text { staff, physio- } \\
\text { therapist, doc- } \\
\text { tor, nurse } \\
\text { - IPS }\end{array}$ & $\begin{array}{ll}\text { - } & \text { Exercise capacity } \\
\text { - } & \text { Echo evaluation } \\
\text { - } & \text { QoL }\end{array}$ \\
\hline $\begin{array}{l}\text { Frederix, } \\
2016 \text { [34] }\end{array}$ & Good & Belgium & $\begin{array}{l}\text { Cost-effective- } \\
\text { ness analysis of } \\
\text { patients with } \\
\text { CR }\end{array}$ & $\begin{array}{l}\text { 24-week web- } \\
\text { based telereha- } \\
\text { bilitation pro- } \\
\text { gram (web- } \\
\text { based coaching, } \\
\text { accelerometer) } \\
\text { plus CR, com- } \\
\text { pared to CR } \\
\text { alone }\end{array}$ & $\begin{array}{ll}- & \mathrm{BA} \\
- & \mathrm{NC} \\
- & \mathrm{TC} \\
- & \mathrm{PAC} \\
- & \mathrm{ET}\end{array}$ & $\begin{array}{ll}\text { - } & \text { Cardiac } \\
\text { nurse, rehabil- } \\
\text { itation nurse } \\
\text { - } \\
\text { IPS }\end{array}$ & $\begin{array}{ll}\text { - } & \text { Cost-effectiveness } \\
\text { - } & \text { Rehospitalizations }\end{array}$ \\
\hline $\begin{array}{l}\text { Skobel, } 2016 \\
{[35]}\end{array}$ & Good & $\begin{array}{ll}\text { - } & \text { Ger- } \\
\text { many } \\
\text { - } & \text { Spain } \\
\text { Unit- } \\
\text { ed } \\
\text { King- } \\
\text { dom }\end{array}$ & $\begin{array}{l}\text { RCT among pa- } \\
\text { tients with CAD } \\
\text { referred for CR }\end{array}$ & $\begin{array}{l}\text { 6-month smart- } \\
\text { phone-based ex- } \\
\text { ercise interven- } \\
\text { tion (remote } \\
\text { monitoring, } \\
\text { physiologic da- } \\
\text { ta capture, and } \\
\text { coaching), as } \\
\text { compared to } \\
\text { conventional } \\
\text { CR }\end{array}$ & $\begin{array}{ll}- & \text { BA } \\
- & \text { PAC } \\
- & \text { ET }\end{array}$ & $\begin{array}{ll}\text { - } & \text { Sports physi- } \\
\text { cians, exer- } \\
\text { cise scientists } \\
\text { - } \quad \text { IPS } \\
\text { - } \quad \text { SAI }\end{array}$ & Exercise capacity \\
\hline $\begin{array}{l}\text { Thorup, } \\
2016 \text { [36] }\end{array}$ & Good & Denmark & $\begin{array}{l}\text { RCT among } \\
\text { hospitalized pa- } \\
\text { tients with } \\
\text { ACS, HF, or } \\
\text { coronary bypass } \\
\text { surgery }\end{array}$ & $\begin{array}{l}\text { 3-month telere- } \\
\text { habilitation trial } \\
\text { with pedometer, } \\
\text { compared } \\
\text { among } 3 \text { rehabil- } \\
\text { itation settings }\end{array}$ & $\begin{array}{ll}\text { - } & \text { BA } \\
- & \mathrm{WM} \\
- & \mathrm{BPM} \\
\text { - } & \mathrm{PAC}\end{array}$ & $\begin{array}{ll}\text { - } & \text { Personal } \\
& \text { nurse } \\
\text { - } & \text { GS } \\
\text { - } & \text { IPS }\end{array}$ & Step count \\
\hline $\begin{array}{l}\text { da Silva } \\
\text { Vieira, } 2017 \\
{[37]}\end{array}$ & Good & Portugal & $\begin{array}{l}\text { RCT among pa- } \\
\text { tients who com- } \\
\text { pleted CR }\end{array}$ & $\begin{array}{l}\text { 6-month virtual } \\
\text { reality CR inter- } \\
\text { vention } \\
\text { (Kinect) or } \\
\text { booklet CR in- } \\
\text { tervention, } \\
\text { compared with } \\
\text { standard of care }\end{array}$ & $\begin{array}{ll}- & \text { BA } \\
- & \text { PAC } \\
- & \text { ET }\end{array}$ & $\begin{array}{ll}\text { - } & \text { Researchers } \\
\text { - } & \text { IPS }\end{array}$ & $\begin{array}{ll}\text { - } & \text { Body composition } \\
\text { - } & \text { Eating patterns } \\
\text { - } & \text { Lipid profile }\end{array}$ \\
\hline
\end{tabular}




\begin{tabular}{|c|c|c|c|c|c|c|c|}
\hline Reference & Quality & Country & $\begin{array}{l}\text { Design and } \\
\text { population }\end{array}$ & Intervention & $\begin{array}{l}\mathrm{CR}^{\mathrm{a}} \\
\text { components delivered }^{\mathrm{b}}\end{array}$ & $\begin{array}{l}\text { Personnel and } \\
\text { delivery setting }^{\mathrm{c}}\end{array}$ & Key outcomes \\
\hline $\begin{array}{l}\text { Hwang, } \\
2017 \text { [38] }\end{array}$ & & & $\begin{array}{l}\text { RCT among } \\
\text { stable patients } \\
\text { with chronic HF }\end{array}$ & $\begin{array}{l}\text { 12-week home- } \\
\text { based CR with } \\
\text { web-based } \\
\text { video conferenc- } \\
\text { ing, compared } \\
\text { with facility- } \\
\text { based CR }\end{array}$ & $\begin{array}{ll}- & \mathrm{BA} \\
- & \mathrm{NC} \\
- & \mathrm{PAC} \\
- & \mathrm{ET} \\
- & \mathrm{PM}\end{array}$ & $\begin{array}{ll}\text { - } & \text { Research } \\
& \text { staff, physio- } \\
\text { therapists } \\
\text { - } & \text { GS } \\
\text { - } & \text { IPS } \\
\text { - } & \text { SAI }\end{array}$ & $\begin{array}{ll}\text { - } & \text { Exercise capacity } \\
\text { - } & \text { QoL }\end{array}$ \\
\hline $\begin{array}{l}\text { Fang, } 2018 \\
{[39]}\end{array}$ & Good & China & $\begin{array}{l}\text { RCT among pa- } \\
\text { tients at post- } \\
\text { PCI }^{\text {ad }}\end{array}$ & $\begin{array}{l}\text { 6-week home- } \\
\text { based CR with } \\
\text { remote physio- } \\
\text { logical monitor- } \\
\text { ing and educa- } \\
\text { tion, as com- } \\
\text { pared with con- } \\
\text { ventional CR }\end{array}$ & $\begin{array}{ll}- & \text { BA } \\
- & \text { PAC } \\
- & \text { ET }\end{array}$ & $\begin{array}{ll}\text { - } & \text { Medical team } \\
\text { - } & \text { IPS }\end{array}$ & $\begin{array}{ll}\text { - } & \text { Exercise capacity } \\
\text { - } & \text { BP } \\
\text { - } & \text { QoL } \\
\text { Nicotine depen- } \\
\text { dence }\end{array}$ \\
\hline $\begin{array}{l}\text { Harzand, } \\
2018[40]\end{array}$ & Good & $\begin{array}{l}\text { United } \\
\text { States }\end{array}$ & $\begin{array}{l}\text { Pilot study of } \\
\text { veterans with } \\
\text { CHD and eligi- } \\
\text { ble for CR }\end{array}$ & $\begin{array}{l}\text { 12-week home- } \\
\text { based CR with } \\
\text { smartphone app } \\
\text { utilizing exer- } \\
\text { cise reminders, } \\
\text { educational ma- } \\
\text { terials, vitals } \\
\text { monitoring, and } \\
\text { remote coach- } \\
\text { ing }\end{array}$ & $\begin{array}{ll}\text { - } & \text { BA } \\
\text { - } & \text { WM } \\
\text { - } & \text { BPM } \\
\text { - } & \text { PAC } \\
\text { - } & \text { ET }\end{array}$ & 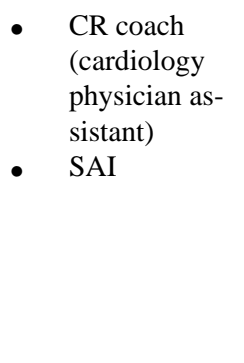 & $\begin{array}{ll}\text { - } & \text { Feasibility } \\
\text { - } & \text { BP } \\
\text { - } & \text { Acceptability } \\
& \text { Exercise capacity }\end{array}$ \\
\hline $\begin{array}{l}\text { Maddison, } \\
2018 \text { [41] }\end{array}$ & Good & $\begin{array}{l}\text { New } \\
\text { Zealand }\end{array}$ & $\begin{array}{l}\text { RCT among pa- } \\
\text { tients with CHD }\end{array}$ & $\begin{array}{l}\text { 12-week remote- } \\
\text { ly monitored } \\
\text { telerehabilita- } \\
\text { tion with coach- } \\
\text { ing, compared } \\
\text { with convention- } \\
\text { al CR }\end{array}$ & $\begin{array}{ll}- & \text { BA } \\
- & \text { PAC } \\
- & \text { ET }\end{array}$ & $\begin{array}{l}\text { - } \quad \text { R exercise } \\
\text { specialist } \\
\text { - } \quad \text { IPS }\end{array}$ & $\begin{array}{ll}\text { - } & \text { Exercise capacity } \\
\text { - } & \text { QoL } \\
\text { - } & \text { Intervention adher- } \\
\text { ence }\end{array}$ \\
\hline $\begin{array}{l}\text { Peng, } 2018 \\
{[42]}\end{array}$ & Good & China & $\begin{array}{l}\text { RCT among pa- } \\
\text { tients with HF }\end{array}$ & $\begin{array}{l}\text { 8-week home- } \\
\text { based CR with } \\
\text { remote coach- } \\
\text { ing using physi- } \\
\text { ologic data cap- } \\
\text { ture, web-based } \\
\text { portal, and } \\
\text { smartphone, } \\
\text { compared with } \\
\text { standard of care }\end{array}$ & $\begin{array}{ll}\text { - } & \text { BA } \\
\text { - } & \text { WM } \\
\text { - } & \text { BPM } \\
\text { - } & \text { PAC } \\
\text { - } & \text { ET } \\
\text { - } & \text { PM }\end{array}$ & $\begin{array}{ll}\text { - } & \text { Multidisci- } \\
\text { plinary CR } \\
\text { team } \\
\text { - } \\
\text { IPS }\end{array}$ & $\begin{array}{ll}\text { - } & \text { Exercise capacity } \\
\text { - } & \text { QoL } \\
\text { - } & \text { Echo evaluation }\end{array}$ \\
\hline $\begin{array}{l}\text { Rawstorn, } \\
2018 \text { [43] }\end{array}$ & Good & $\begin{array}{l}\text { New } \\
\text { Zealand }\end{array}$ & $\begin{array}{l}\text { RCT among pa- } \\
\text { tients with } \mathrm{CHD} \\
\text { eligible for CR }\end{array}$ & $\begin{array}{l}\text { 12-week remote- } \\
\text { ly monitored } \\
\text { telerehabilita- } \\
\text { tion with coach- } \\
\text { ing, as com- } \\
\text { pared with con- } \\
\text { ventional CR }\end{array}$ & $\begin{array}{ll}- & \text { BA } \\
- & \text { PAC } \\
- & \text { ET }\end{array}$ & Exercise specialist & $\begin{array}{ll}\text { - } & \text { Usability } \\
\text { - } & \text { Satisfaction }\end{array}$ \\
\hline
\end{tabular}

\footnotetext{
${ }^{\mathrm{a}} \mathrm{CR}$ : cardiac rehabilitation.

${ }^{\mathrm{d}} \mathrm{ACS}$ : acute coronary syndrome.

${ }^{\mathrm{e}} \mathrm{BA}$ : baseline assessment.

f PAC: physical activity counseling.

${ }^{\mathrm{g}}$ ET: exercise training.

${ }^{h}$ IPS: in-person session.

${ }^{\mathrm{i}} \mathrm{SAI}$ : standalone intervention.

${ }^{j}$ QoL: quality of life.
}

${ }^{\mathrm{b}} \mathrm{CR}$ components were delivered through digital interventions except for baseline assessment that were conducted in person.

${ }^{\mathrm{c}}$ Delivery setting: group sessions (GS), in-person session (IPS), standalone intervention (SAI). 
${ }^{k}$ RCT: randomized controlled trial.

${ }^{\mathrm{l}} \mathrm{CAD}$ : coronary artery disease.

$\mathrm{m}_{\mathrm{NC}}$ : nutrition counseling.

${ }^{\mathrm{n}} \mathrm{WM}$ : weight management.

${ }^{\circ} \mathrm{LM}$ : lipid management.

$\mathrm{p}_{\mathrm{TC}}$ : tobacco cessation.

qPM: psychological management.

${ }^{\mathrm{r}} \mathrm{CVD}$ : cardiovascular disease.

${ }^{\mathrm{s}} \mathrm{CABG}$ : coronary artery bypass grafting.

${ }^{\mathrm{t}} \mathrm{ECG}$ : electrocardiogram.

${ }^{\mathrm{u}} \mathrm{GS}$ : group session.

${ }^{\mathrm{v}} \mathrm{HF}$ : heart failure.

${ }^{\mathrm{w}}$ BPM: blood pressure management.

${ }^{\mathrm{x}}$ MI: myocardial infarction.

${ }^{\mathrm{y}} \mathrm{BP}$ : blood pressure.

${ }^{\mathrm{z}} \mathrm{DM}$ : diabetes management.

${ }^{\mathrm{aa}} \mathrm{CHD}$ : coronary heart disease.

${ }^{\mathrm{ab}}$ IHD: ischemic heart disease.

${ }^{\mathrm{ac}}$ CRT-D: cardiac resynchronization therapy with defibrillator function.

${ }^{\mathrm{ad}} \mathrm{PCI}$ : percutaneous coronary intervention.

Table 2. Summary of the studies included in the analysis $(n=31)$.

\begin{tabular}{|c|c|}
\hline Characteristics & Value \\
\hline \multicolumn{2}{|l|}{ Type of study, n (\%) } \\
\hline $\mathrm{RCT}^{\mathrm{a}}$ & $22(71)$ \\
\hline Pilot study & $5(16)$ \\
\hline Nonrandomized trial & $2(6)$ \\
\hline Cost-effectiveness analysis from RCT & $1(3)$ \\
\hline Qualitative study & $1(3)$ \\
\hline \multicolumn{2}{|l|}{ Location of study, by continent, $n(\%)$} \\
\hline Europe & $12(39)$ \\
\hline North America & $8(26)$ \\
\hline Australia (including New Zealand) & $8(26)$ \\
\hline Asia & $3(10)$ \\
\hline \multicolumn{2}{|l|}{ Publication year, n (\%) } \\
\hline $1990-2000$ & $1(3)$ \\
\hline $2001-2010$ & $6(19)$ \\
\hline 2011-2018 & $24(77)$ \\
\hline Median sample size (IQR) & $98(52.5-146)$ \\
\hline Median follow-up time (months; IQR) & $6(3-6)$ \\
\hline Median intervention duration (months; IQR) & $3(1.6-4.4)$ \\
\hline
\end{tabular}

${ }^{\mathrm{a}} \mathrm{RCT}$ : randomized controlled trial. 
Figure 2. Percentage of studies with (1) the cardiac rehabilitation (CR) core components being addressed through digital interventions (apart from baseline assessment, which was conducted in person), (2) the technology modalities used in digital CR interventions, (3) the types of personnel employed in each CR program, and (4) the key outcomes evaluated. Cumulative percentages in some instances exceed $100 \%$ because some studies looked at multiple traits $(n=31)$. BP: blood pressure; CR: cardiac rehabilitation; ECG: electrocardiogram; HR: heart rate; PT: physical therapist.

\section{CR core components}

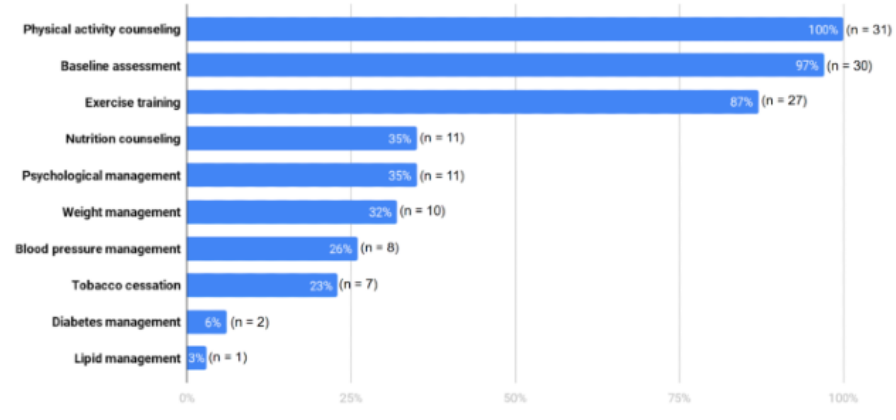

Personnel

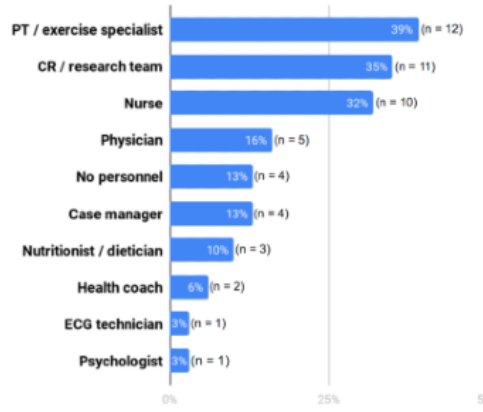

\section{Key Outcomes}

The key findings from the 31 studies are summarized in Textbox 2. Overall, program adherence was greater in patients using digital interventions than in those participating in conventional CR. Moreover, digital CR interventions were comparable to conventional CR (control) groups across multiple short-term outcomes (eg, functional capacity, physical activity, self-efficacy, program adherence, weight management, dietary habits, and QoL). However, digital CR interventions had mixed efficacy with regard to blood pressure control and mood. Of the
Digital CR technology modalities

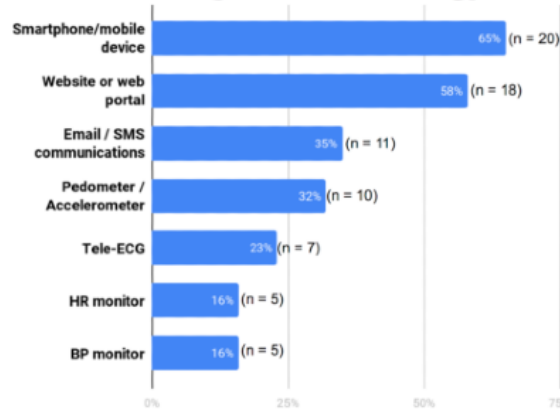

Key outcomes

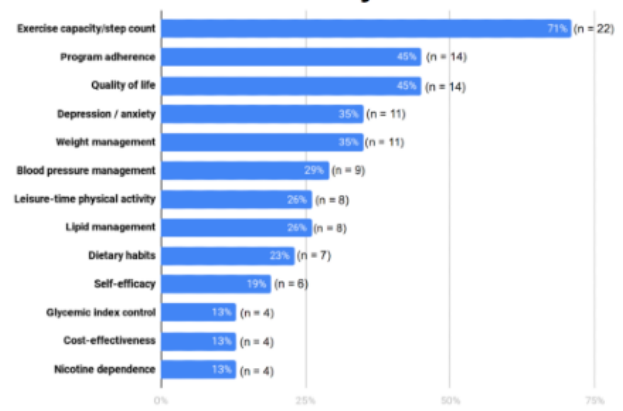

9 studies reporting blood pressure as an outcome of digital interventions versus conventional $\mathrm{CR}, 3$ studies showed noninferiority [18,29,41], 4 found no significant impact on blood pressure [19,28,31,39], and 2 reported a better control of blood pressure in the control group as compared to the digital intervention group [25,35]. Similarly, although the majority of studies assessing mood as an outcome reported that digital interventions were noninferior to conventional $\mathrm{CR}$ in improving mood [22,25,29,30,33], 4 studies reported no significant improvement in anxiety or depression [19,24,25,42]. 
Textbox 2. Summary of findings by thematic outcomes.

Blood glucose control

- Only 1 of the 4 studies reported an improvement in glycemic control in the intervention group [31]. In total, 2 studies found no significant impact of the digital cardiac rehabilitation (CR) intervention on glycemic control [29,35]. One study found no significant difference in glycemic control between the digital intervention and usual care groups [41].

Blood pressure management

- In total, 3 of the 9 studies described CR interventions that significantly improved blood pressure management and were noninferior to the control groups $[18,29,41]$. A total of 4 studies did not find the digital CR interventions to significantly influence blood pressure management [19,28,31,39]. In all, 2 studies found that the control group had better blood pressure management as compared to the intervention group [25,35].

Depression or anxiety

- In total, 5 studies reported a positive effect on mood in the intervention group [22,25,29,30,33]. A total of 4 studies found that digital interventions had no significant impact on mood [19,24,25,42]. In total, 2 studies found no significant change in the psychological status between digital intervention and usual care groups [35,39]. One study found that both the intervention and usual care groups experienced an improvement in mood as compared with baseline, and there was no significant difference between the intervention and control groups [39]. One study found a negative effect on depression in the intervention group [16]. Of note, Devi et al [25] found that mood improved in the short term but was not significantly different from baseline at 6 months.

Dietary habits

- In total, 5 of the 7 studies found improvement in dietary habits [24,29,30,32,37], whereas 2 studies found that the intervention had no significant impact on the participants' dietary habits $[19,25]$.

Exercise capacity

- In total, 22 studies looked at functional capacity as an outcome, and all of them reported that the intervention group was not inferior to the control group [15,17-22,24,25,27-33,35,38-42]. Of note, 4 studies found no significant difference in the functional capacity between the digital intervention and usual care groups [19,31,33,41]. Furthermore, Smolis-Bak et al [33] found functional capacity at the 12-month follow-up to be comparable to that at the baseline visit.

Lipid management

- In total, 2 of the 8 studies reported an increase in high-density lipoprotein in the intervention group [31,37], 2 of the 8 reported decreased low-density lipoprotein and total cholesterol [18,32], and 2 of the 8 studies reported a decrease in triglycerides $[29,30]$.

Nicotine dependence

- Only 1 [39] of the 4 studies showed an improvement in smoking habits as measured by the Fagerstrom Test for Nicotine Dependence score $[18,19,24,39]$.

Physical activity

- All 8 studies examining physical activity found that there was an improvement in the intervention group, comparable to or even greater than the increase in the physical activity in the control group [14,15,22,25,31,32,36,41].

Program adherence

- All 14 studies evaluating program adherence to CR reported that adherence to digital interventions was not inferior to traditional interventions [19-21,26-30,35,37,38,40,41,43]. Of note, 9 of the 14 studies found adherence to be greater in the digital intervention group $[20,21,26,28-30,35,38,40]$.

Quality of life (QoL)

- In total, 10 studies reported an improvement in QoL in the intervention group [15,17,22,24,25,27,29,33,39,42]. A total of 6 studies found no significant difference in QoL measures between the digital intervention and usual care groups [17,21,27,35,38,41].

Self-efficacy

- Of the 6 studies evaluating self-efficacy, 5 showed improvement in self-efficacy following the digital interventions [13,15,25,32,41]. Pfaeffli Dale et al [16] found that the digital CR intervention had no significant impact on the participants' overall self-efficacy.

Weight management

- Digital CR interventions effectively addressed weight management in 8 of the 11 studies [18,19,25,28-30,37,41]. A total of 2 studies found no difference in weight or body mass index before and after the digital CR intervention [24,35]. In one study [17], the home group had increased weight, whereas the on-site control group had a slightly decreased weight. 
We found a paucity of studies specifying intervention components that targeted lipid management, glycemic index control, and smoking cessation. These components were often reported as secondary outcome measures, if at all. Furthermore, the research on the long-term efficacy of digital CR interventions was sparse with heterogeneous findings. Although Devi et al [25] found improvement in outcomes such as QoL, self-efficacy, and physical activity in the short term, no significant intervention effect was present on these outcomes when assessed at the 6-month follow-up. However, they noted that the intervention group demonstrated trends of improved levels of physical activity, whereas the control group did not. Reid et al [22] also reported long-term improvements in self-reported QoL and physical activity as long as 12 months from the start of the digital CR program, which was delivered over a 6-month period.

The examination of the studies by follow-up time revealed that the majority of the key outcome findings were mixed. However, all the studies reporting outcomes regarding adherence [19-21,26-30,35,37,38,40,41,43],

QoL $[15,17,21,22,24,25,27,29,33,35,38,39,41,42]$, and exercise capacity $[15,17-22,24,25,27-33,35,38-42]$ found positive results or outcomes that were noninferior to the control group. Only studies with a follow-up period longer than 3 months reported outcomes for physical activity [14,15,22,25,31,32,36,41] and blood glucose control [29,31,35,41]. Regarding physical activity, the results were positive [14,15,22,25,31,32,36,41], whereas the results regarding blood glucose management were mixed, with positive effects [31], nonsignificant effects [29,35], or comparable results between the intervention and the control groups [41]. Similarly, the outcomes for blood pressure, depression or anxiety, and weight management were mixed (with positive effects, nonsignificant effects, or comparable results between the intervention and control groups) in both studies with shorter (3 months or less) [18,24,28,39] and longer (more than 3 months) [19,22,25,29-31,33,35,37,41,42] follow-up times. The only exceptions were as follows: one study reported a negative impact on mood at 6 months [16], 2 studies reported that the control group had better blood pressure management than the intervention group [25,35], and one study found an increase in body weight in the intervention group at 3 months [17]. For studies reporting outcomes regarding dietary habits, lipid management, and self-efficacy, studies with longer than a 3-month follow-up period reported positive or nonsignificant effects $[15,16,19,25,29-32,35,37,41]$ or outcomes that were comparable between the intervention and the control groups $[35,41]$, whereas studies with a follow-up period of 3 months or less found positive results [13,18,24]. The outcomes for nicotine dependence were mixed (positive or nonsignificant effects) among the studies with a short follow-up period $[18,24,39]$, whereas one study reported no impact on smoking at 6 months [19].

The examination of more comprehensive, standalone interventions revealed that only 6 studies included in this review were standalone interventions delivering 5 or more $\mathrm{CR}$ components (other than a baseline assessment) [21,23-25,29,32]. The results of these studies for the majority of the key outcomes related to the $\mathrm{CR}$ components delivered through the interventions were heterogeneous. Of these 6 more comprehensive standalone interventions, only Brough et al [24] reported the outcomes regarding nicotine dependence, finding no impact on smoking. Devi et al [25] and Brough et al [24] found that their digital interventions had no significant impact on mood, whereas Varnfield et al [29] reported a positive effect on mood and anxiety levels in the intervention groups. Regarding dietary habits, Varnfield et al [29], Lear et al [32], and Brough et al [24] found improvement in the dietary habits of the intervention group, whereas Devi et al [25] found no significant impact. Three of these studies [24,25,29] reported an improvement in QoL in the intervention group, but Piotrowicz et al [21] found no significant difference in QoL measures between the intervention and control groups. Five of these studies reported a positive effect of digital intervention on exercise capacity [21,24,25,29,32]. In terms of weight management, Devi et al [25] and Varnfield et al [29] found a positive impact on weight in the intervention group, whereas Brough et al [24] found no impact on weight in the intervention group. Overall, there was a wide variety in the interventions delivered and outcomes reported.

\section{Discussion}

\section{The Potential of Digital CR}

This study highlights digital technology as a potential means of enhancing care and broadening access to CR through tailored interactive interventions. Our work differs from previous systematic reviews as our emphasis is on digital CR interventions with a focus on providing a systematic evaluation of the current literature to better understand the characteristics of these interventions. This study builds upon a growing body of literature supporting the use of internet-based features such as web portals and digital devices (eg, wearables) to remotely deliver CR components.

We found that digital CR was feasible and as effective as traditional CR in improving outcomes, whether as an adjunct or as an alternative to traditional CR [16,21,26,29,39,41,42]. Our findings support the conclusions of a previous study demonstrating a similar effectiveness of home- and center-based $\mathrm{CR}$ in improving clinical and health-related QoL outcomes in patients with myocardial infarction, myocardial revascularization, and heart failure [44]. In addition, Huang et al found that telehealth $\mathrm{CR}$ interventions were noninferior to center-based CR, both in the short term (12 weeks-1 year) and long term (up to 6 years), when comparing participants' exercise capacity, all-cause mortality, and modifiable risk factors, including blood pressure, blood lipids, smoking, and weight [45]. Moreover, the AACVPR, AHA, and ACC recently released a consensus statement highlighting evidence that home- and facility-based CR can achieve similar improvements in 3-12-month clinical outcomes [10]. These developments highlight digital technology as a potential means of enhancing care and broadening access to CR through tailored interactive interventions $[46,47]$. However, our study and literature indicate that until digital CR is further developed and better understood, there will be a need for in-person CR sessions (Multimedia Appendix 6). In-person sessions may help digital CR by establishing baseline and monitoring progress, personalizing 
treatment plans, and bridging patient technology-usage challenges through technology education and deployment, especially for older users [48-50]. Currently, there are several ongoing clinical trials that are studying the efficacy of digital CR interventions [51-54].

\section{Key Recommendations for Future Research}

A limitation of our study is the heterogeneity of the identified papers, thus prohibiting meta-analysis. Papers varied in CR technologies, interventions, study design, measured outcomes, and control groups. For example, when considering a traditional
CR population as a control group, some studies used standard care, some used direct comparison with facility-based CR, and other feasibility studies did not have a control comparison group. Although the diversity in studies proved challenging to quantify, it reiterates the motivation of this systematic review: digital health-based $\mathrm{CR}$ is emerging as an alternative or adjunct to standard CR; thus, methodologies have yet to reach a consensus. Consequently, we encourage practitioners to study digital CR approaches in a collaborative environment to promote the standardization and optimization of study methods. We have summarized our key recommendations in Textbox 3.

Textbox 3. Key recommendations for researchers conducting digital cardiac rehabilitation studies.

- Clearly state the specific goals of the intervention: whether it is designed to be a comprehensive standalone program or to be used adjunctively with traditional cardiac rehabilitation $(\mathrm{CR})$

- Describe the specifics of the CR components targeted through the intervention and the technology, equipment, or personnel required to deliver each of the components

- Include the specific details of the comparison group (eg, elaborate on what usual care consists of and include information about the specific intervention the comparison group received)

Beyond the heterogeneity of the study designs, we identified other limitations. Although we evaluated CR program components using the AHA and AACVPR's consensus statement on the core components of CR, the CR programs outside of the United States may differ from professional society guidelines in the United States. In addition, some studies included only minimal details about the specific components of their interventions; thus, the number of CR components delivered may have been greater than that captured in this review. Although studies reported the technology used (summarized in Multimedia Appendix 7), specific details were often sparse, limiting the evaluation of specifically what worked well and what was difficult to implement. Furthermore, the studies did not address long-term outcomes, as the maximum reported follow-up was 16 months, and most studies had fairly small sample sizes. Finally, the majority of the studies reviewed included sessions that were conducted in person for at least a portion of the overall CR intervention.

We identified several potential directions for future research. Although digital interventions have been found to successfully deliver the components of CR pertaining to physical activity, there remains a paucity of comprehensive digital CR program interventions that address risk factors such as lipid management, blood glucose level control, and smoking cessation. In addition, the long-term effectiveness of digital approaches to CR requires further evaluation [8,55]. Furthermore, studies with larger sample sizes and adequate control groups for comparison are necessary to better understand the impact of these interventions. Additional studies are also required to investigate the frequency of adverse events in patients participating in these interventions compared with traditional approaches. The adverse effects reported in the studies reviewed fell into the categories of (1) cardiac related (acute coronary syndrome, stable angina, arrhythmias, pericarditis, dyspnea, syncope, etc), (2) potential cardiac etiology (pleural effusions, cerebral ischemia, etc), (3) noncardiac related (peripheral artery disease, pneumonia, accidents, etc), and (4) death. A number of studies reported no significant difference in the rate of adverse effects between the digital intervention and control groups [14,30,33,38]. There were also studies that found that the intervention group had fewer associated adverse events than the control group; however, this did not always reach statistical significance $[19,22,31,32,34]$. Only one study reported that the digital CR intervention group had more adverse events during the treatment period as compared with the control group that participated in a center-based CR; however, adverse events were comparable during the postintervention follow-up period [41]. In some cases, no major adverse events occurred throughout the study $[17,26-28,40,42]$, or the adverse effects were unrelated to the study intervention $[16,21,35]$. No conclusions could be drawn regarding the increased or decreased risk of adverse effects in some studies $[15,18,20]$. Overall, our findings highlight the need for robust digital intervention study designs with more comprehensive programs and analysis of both the short- and long-term effects.

Furthermore, as briefly mentioned in the Methods section, our team consulted with an external expert to include relevant original research on digital approaches to $\mathrm{CR}$ that may have been inadvertently excluded from our primary search strategy. We opted for this approach given that although the field of telehealth and telemedicine has grown rapidly over the past few decades, the adoption of common terminology remains in infancy [7]. The evolution of this terminology has been demonstrated in a bibliometric analysis by Fatehi and Wootton [6], who noted the emergence of terms such as eHealth and mHealth as well as the usage trends of the terms such as telemedicine, telehealth, and eHealth in the literature. As a result, there may be studies that include digital components, such as the usage of the internet, which our search terms failed to capture. Our experience further highlights the importance of more standardized terminology surrounding digital health interventions and an understanding of the evolving terminology to accurately review the existing literature. 


\section{Conclusions}

Overall, we found that digital technology offers the potential to address the challenges associated with traditional, facility-based CR. If implemented on a large scale, digital CR could provide a level of impact, accessibility, affordability, cost savings, and benefits to patients not possible with conventional CR. However, so far, interventions have primarily focused on physical activity counseling and exercise training and not on the other core components of CR. In addition, our study focused on the evaluation of the technology used in digital CR and the comprehensiveness of these programs, but the intricacies of accreditation for CR programs are beyond the scope of this review. Further research is required with more comprehensive $\mathrm{CR}$ interventions to understand the long-term clinical impact of digital CR solutions on key cardiovascular outcomes and establish best practices for the development, delivery, and assessment of digital CR.

\section{Acknowledgments}

The authors thank Julie Nanavati, MLS, MA, (Johns Hopkins Welch Medical Library Informationist) for her assistance in developing the search strategy and downloading the references.

SW is supported by the Johns Hopkins School of Medicine Medical Scientist Training Program (National Institutes of Health: Institutional Predoctoral Training Grant - 5T32GM007309) and the National Institutes of Health: Ruth L. Kirschstein Individual Predoctoral NRSA for MD/PhD: F30 Training Grant (F30HL142131). ES is supported by the National Institutes of Health (NIH)/National Institute of Nursing Research (NINR) F31 NR017328, Ruth L. Kirschstein National Research Service Award and NIH/NINR T32 NR012704, predoctoral fellowship in interdisciplinary cardiovascular health research. KR was supported by grant TL1 TR003100 from the National Center for Advancing Translational Sciences, NIH. Funding sources for this project had no role in the design or conduct of the study or in data collection, management, or analysis, manuscript preparation, or in decisions about submitting the manuscript for publication. SM receives research support from the Aetna Foundation, the American Heart Association (20SFRN35380046 and COVID19-811000), the David and June Trone Family Foundation, the Pollin Digital Innovation Fund, Google, Patient-Centered Outcomes Research Institute (ME-2019C1-15328), the National Institutes of Health (P01 HL108800), Nokia, and the PJ Schafer Memorial Fund.

\section{Authors' Contributions}

SW, FM, and SM contributed substantially to the conception and design of this work. SW, RS, KR, and JW screened the references for inclusion in the review. NG provided external expert review on article selection. SW, EH, PH, HX, RS, KR, JW, and LS performed data extraction and analysis. YG and NO reviewed the studies to determine study quality. SW, EH, PH, HX, RS, KR, JW, YG, NO, LS, ES, GH, KK, WY, FM, EL, DM, NG, and SM provided a critical review of the manuscript.

\section{Conflicts of Interest}

FM and SM are founders of and hold equity in Corrie Health, which intends to further develop the digital platform. This arrangement has been reviewed and approved by the Johns Hopkins University in accordance with its conflicts of interest policies. Outside of the present work, they have received material support from Apple and iHealth and funding from the Maryland Innovation Initiative, Wallace H. Coulter Translational Research Partnership, Louis B. Thalheimer Fund, the Johns Hopkins Individualized Health Initiative, and the American Heart Association. SM reports personal fees for serving on scientific advisory boards for Akcea Therapeutics, Amgen, AstraZeneca, DalCor Pharmaceuticals, Esperion, Novo Nordisk, Quest Diagnostics, Regeneron, Sanofi, and 89bio. SM is a coinventor with a pending patent filed by the Johns Hopkins University for a system of low-density lipoprotein cholesterol estimation. EL is a scientific advisor for Moving Analytics. NG is the managing member of a population health management company, INTERVENT International, LLC.

\section{Multimedia Appendix 1}

Search Terms.

[DOCX File, 15 KB-Multimedia Appendix 1]

\section{Multimedia Appendix 2}

National Institutes of Health quality assessment tools: quality assessment of controlled intervention studies.

[DOCX File, 15 KB-Multimedia Appendix 2]

\section{Multimedia Appendix 3}

National Institutes of Health quality assessment tools: quality assessment tool for observational cohort and cross-sectional studies. [DOCX File, 15 KB-Multimedia Appendix 3] 


\section{Multimedia Appendix 4}

Summary of study and patient characteristics.

[DOCX File, 24 KB-Multimedia Appendix 4]

\section{Multimedia Appendix 5}

National Institutes of Health quality assessment for studies reviewed.

[DOCX File , $41 \mathrm{~KB}-$ Multimedia Appendix 5]

\section{Multimedia Appendix 6}

Aspects of in-person sessions.

[DOCX File, 19 KB-Multimedia Appendix 6]

\section{Multimedia Appendix 7}

Technology used in studies.

[DOCX File, 18 KB-Multimedia Appendix 7]

\section{References}

1. Cardiovascular disease. WHO. 2019. URL: http://www.who.int/cardiovascular diseases/about cvd/en/ [accessed 2020-12-29]

2. Anderson L, Oldridge N, Thompson DR, Zwisler A, Rees K, Martin N, et al. Exercise-based cardiac rehabilitation for coronary heart disease: Cochrane systematic review and meta-analysis. J Am Coll Cardiol 2016 Jan 05;67(1):1-12 [FREE Full text] [doi: 10.1016/j.jacc.2015.10.044] [Medline: 26764059]

3. Balady GJ, Williams MA, Ades PA, Bittner V, Comoss P, Foody JM, American Heart Association Exercise, Cardiac Rehabilitation, Prevention Committee, the Council on Clinical Cardiology, American Heart Association Council on Cardiovascular Nursing, American Heart Association Council on Epidemiology Prevention, American Heart Association Council on Nutrition, Physical Activity, Metabolism, American Association of Cardiovascular Pulmonary Rehabilitation. Core components of cardiac rehabilitation/secondary prevention programs: 2007 update: a scientific statement from the American Heart Association Exercise, Cardiac Rehabilitation, and Prevention Committee, the Council on Clinical Cardiology; the Councils on Cardiovascular Nursing, Epidemiology and Prevention, and Nutrition, Physical Activity, and Metabolism; and the American Association of Cardiovascular and Pulmonary Rehabilitation. Circulation 2007 May 22;115(20):2675-2682. [doi: 10.1161/CIRCULATIONAHA.106.180945] [Medline: 17513578]

4. Boyden T, Rubenfire M, Franklin B. Will increasing referral to cardiac rehabilitation improve participation? Prev Cardiol 2010;13(4):192-201 [FREE Full text] [doi: 10.1111/j.1751-7141.2010.00086.x] [Medline: 20879184]

5. Balady GJ, Ades PA, Bittner VA, Franklin BA, Gordon NF, Thomas RJ, American Heart Association Science Advisory Coordinating Committee. Referral, enrollment, and delivery of cardiac rehabilitation/secondary prevention programs at clinical centers and beyond: a presidential advisory from the American Heart Association. Circulation 2011 Dec 20;124(25):2951-2960. [doi: 10.1161/CIR.0b013e31823b21e2] [Medline: 22082676]

6. Fatehi F, Wootton R. Telemedicine, telehealth or e-health? A bibliometric analysis of the trends in the use of these terms. J Telemed Telecare 2012 Dec;18(8):460-464. [doi: 10.1258/jtt.2012.gth108] [Medline: 23209265]

7. Scott RE, Mars M. The same language speak we do - consensus terminology for telehealth. Stud Health Technol Inform 2016;231:99-109. [Medline: 27782021]

8. Bhavnani SP, Parakh K, Atreja A, Druz R, Graham GN, Hayek SS, et al. 2017 roadmap for innovation-ACC health policy statement on healthcare transformation in the era of digital health, big data, and precision health: a report of the American College of Cardiology task force on health policy statements and systems of care. J Am Coll Cardiol 2017 Nov 28;70(21):2696-2718 [REE Full text] [doi: 10.1016/j.jacc.2017.10.018] [Medline: 29169478]

9. Sharma A, Harrington RA, McClellan MB, Turakhia MP, Eapen ZJ, Steinhubl S, et al. Using digital health technology to better generate evidence and deliver evidence-based care. J Am Coll Cardiol 2018 Jun 12;71(23):2680-2690 [FREE Full text] [doi: 10.1016/j.jacc.2018.03.523] [Medline: 29880129]

10. Thomas RJ, Beatty AL, Beckie TM, Brewer LC, Brown TM, Forman DE, et al. Home-based cardiac rehabilitation: a scientific statement from the American Association of Cardiovascular and Pulmonary Rehabilitation, the American Heart Association, and the American College of Cardiology. J Am Coll Cardiol 2019 Jul 09;74(1):133-153 [FREE Full text] [doi: 10.1016/j.jacc.2019.03.008] [Medline: $\underline{31097258]}$

11. Study Quality Assessment Tools. National Heart, Lung, and Blood Institute. -. URL: https://www.nhlbi.nih.gov/health-topics/ study-quality-assessment-tools [accessed 2020-12-29]

12. Moher D, Liberati A, Tetzlaff J, Altman DG, PRISMA Group. Preferred reporting items for systematic reviews and meta-analyses: the PRISMA statement. Ann Intern Med 2009 Aug 18;151(4):264-9, W64 [FREE Full text] [doi: 10.7326/0003-4819-151-4-200908180-00135] [Medline: 19622511] 
13. Jenny NYY, Fai TS. Evaluating the effectiveness of an interactive multimedia computer-based patient education program in cardiac rehabilitation. The Occupational Therapy Journal of Research 2001 Aug 24;21(4):260-275. [doi: $10.1177 / 153944920102100404]$

14. Barnason S, Zimmerman L, Nieveen J, Schulz P, Miller C, Hertzog M, et al. Influence of a symptom management telehealth intervention on older adults' early recovery outcomes after coronary artery bypass surgery. Heart Lung 2009;38(5):364-376 [FREE Full text] [doi: 10.1016/j.hrtlng.2009.01.005] [Medline: 19755186]

15. Maddison R, Pfaeffli L, Whittaker R, Stewart R, Kerr A, Jiang Y, et al. A mobile phone intervention increases physical activity in people with cardiovascular disease: results from the HEART randomized controlled trial. Eur J Prev Cardiol 2015 Jun;22(6):701-709. [doi: 10.1177/2047487314535076] [Medline: 24817694]

16. Pfaeffli Dale L, Whittaker R, Jiang Y, Stewart R, Rolleston A, Maddison R. Text message and internet support for coronary heart disease self-management: results from the text4heart randomized controlled trial. J Med Internet Res 2015 Oct 21;17(10):e237 [FREE Full text] [doi: 10.2196/jmir.4944] [Medline: 26490012]

17. Ades PA, Pashkow FJ, Fletcher G, Pina IL, Zohman LR, Nestor JR. A controlled trial of cardiac rehabilitation in the home setting using electrocardiographic and voice transtelephonic monitoring. Am Heart J 2000 Mar;139(3):543-548. [doi: 10.1016/s0002-8703(00)90100-5] [Medline: 10689271]

18. Gordon NF, English CD, Contractor AS, Salmon RD, Leighton RF, Franklin BA, et al. Effectiveness of three models for comprehensive cardiovascular disease risk reduction. Am J Cardiol 2002 Jun 01;89(11):1263-1268. [doi:

10.1016/s0002-9149(02)02323-8] [Medline: 12031725]

19. Southard BH, Southard DR, Nuckolls J. Clinical trial of an Internet-based case management system for secondary prevention of heart disease. J Cardiopulm Rehabil 2003;23(5):341-348. [doi: 10.1097/00008483-200309000-00003] [Medline: 14512778]

20. Scalvini S, Zanelli E, Comini L, Tomba MD, Troise G, Giordano A. Home-based exercise rehabilitation with telemedicine following cardiac surgery. J Telemed Telecare 2009;15(6):297-301. [doi: 10.1258/jtt.2009.090208] [Medline: 19720767]

21. Piotrowicz E, Baranowski R, Bilinska M, Stepnowska M, Piotrowska M, Wójcik A, et al. A new model of home-based telemonitored cardiac rehabilitation in patients with heart failure: effectiveness, quality of life, and adherence. Eur J Heart Fail 2010 Feb;12(2):164-171 [FREE Full text] [doi: 10.1093/eurjhf/hfp181] [Medline: 20042423]

22. Reid RD, Morrin LI, Beaton LJ, Papadakis S, Kocourek J, McDonnell L, et al. Randomized trial of an internet-based computer-tailored expert system for physical activity in patients with heart disease. Eur J Prev Cardiol 2012 Dec;19(6):1357-1354. [doi: 10.1177/1741826711422988] [Medline: 21903744]

23. Clark RA, Tideman P, Tirimacco R, Wanguhu K, Poulsen V, Simpson P, et al. A pilot study of the feasibility of an internet-based electronic Outpatient Cardiac Rehabilitation (eOCR) program in rural primary care. Heart Lung Circ 2013 May;22(5):352-359. [doi: 10.1016/j.hlc.2012.11.005] [Medline: 23294762]

24. Brough C, Boyce S, Houchen-Wolloff L, Sewell L, Singh S. Evaluating the interactive web-based program, activate your heart, for cardiac rehabilitation patients: a pilot study. J Med Internet Res 2014 Oct 29;16(10):e242 [FREE Full text] [doi: 10.2196/jmir.3027] [Medline: 25359204]

25. Devi R, Powell J, Singh S. A web-based program improves physical activity outcomes in a primary care angina population: randomized controlled trial. J Med Internet Res 2014 Sep 12;16(9):e186 [FREE Full text] [doi: 10.2196/jmir.3340] [Medline: 25217464]

26. Forman DE, LaFond K, Panch T, Allsup K, Manning K, Sattelmair J. Utility and efficacy of a smartphone application to enhance the learning and behavior goals of traditional cardiac rehabilitation: a feasibility study. J Cardiopulm Rehabil Prev 2014;34(5):327-334. [doi: 10.1097/HCR.0000000000000058] [Medline: 24866355]

27. Kraal JJ, Peek N, Van den Akker-Van Marle ME, Kemps HM. Effects of home-based training with telemonitoring guidance in low to moderate risk patients entering cardiac rehabilitation: short-term results of the FIT@ Home study. Eur J Prev Cardiol 2014 Nov;21(2 Suppl):26-31 [FREE Full text] [doi: 10.1177/2047487314552606] [Medline: 25354951]

28. Piotrowicz E, Korzeniowska-Kubacka I, Chrapowicka A, Wolszakiewicz J, Dobraszkiewicz-Wasilewska B, Batogowski M, et al. Feasibility of home-based cardiac telerehabilitation: results of TeleInterMed study. Cardiol J 2014;21(5):539-536. [doi: 10.5603/CJ.a2014.0005] [Medline: 24526507]

29. Varnfield M, Karunanithi M, Lee C, Honeyman E, Arnold D, Ding H, et al. Smartphone-based home care model improved use of cardiac rehabilitation in postmyocardial infarction patients: results from a randomised controlled trial. Heart 2014 Nov;100(22):1770-1779 [FREE Full text] [doi: 10.1136/heartjnl-2014-305783] [Medline: 24973083]

30. Whittaker F, Wade V. The costs and benefits of technology-enabled, home-based cardiac rehabilitation measured in a randomised controlled trial. J Telemed Telecare 2014 Oct;20(7):419-422. [doi: 10.1177/1357633X14552376] [Medline: 25400004]

31. Frederix I, Van Driessche N, Hansen D, Berger J, Bonne K, Alders T, et al. Increasing the medium-term clinical benefits of hospital-based cardiac rehabilitation by physical activity telemonitoring in coronary artery disease patients. Eur J Prev Cardiol 2015 Feb;22(2):150-158. [doi: 10.1177/2047487313514018] [Medline: 24249840]

32. Lear SA, Singer J, Banner-Lukaris D, Horvat D, Park JE, Bates J, et al. Improving access to cardiac rehabilitation using the internet: a randomized trial. Stud Health Technol Inform 2015;209:58-66. [Medline: 25980706]

33. Smolis-Bąk E, Dąbrowski R, Piotrowicz E, Chwyczko T, Dobraszkiewicz-Wasilewska B, Kowalik I, et al. Hospital-based and telemonitoring guided home-based training programs: effects on exercise tolerance and quality of life in patients with 
heart failure (NYHA class III) and cardiac resynchronization therapy. A randomized, prospective observation. Int J Cardiol 2015 Nov 15;199:442-447. [doi: 10.1016/j.ijcard.2015.07.041] [Medline: 26276068]

34. Frederix I, Hansen D, Coninx K, Vandervoort P, Vandijck D, Hens N, et al. Effect of comprehensive cardiac telerehabilitation on one-year cardiovascular rehospitalization rate, medical costs and quality of life: a cost-effectiveness analysis. Eur J Prev Cardiol 2016 May;23(7):674-682. [doi: 10.1177/2047487315602257] [Medline: 26289723]

35. Skobel E, Knackstedt C, Martinez-Romero A, Salvi D, Vera-Munoz C, Napp A, et al. Internet-based training of coronary artery patients: the Heart Cycle Trial. Heart Vessels 2017 Apr;32(4):408-418. [doi: 10.1007/s00380-016-0897-8] [Medline: 27730298]

36. Thorup C, Hansen J, Grønkjær M, Andreasen JJ, Nielsen G, Sørensen EE, et al. Cardiac patients' walking activity determined by a step counter in cardiac telerehabilitation: data from the intervention arm of a randomized controlled trial. J Med Internet Res 2016 Apr 04;18(4):e69 [FREE Full text] [doi: 10.2196/jmir.5191] [Medline: 27044310]

37. da Silva Vieira AS, Melo C, Machado J, Gabriel J. The effect of virtual reality on a home-based cardiac rehabilitation program on body composition, lipid profile and eating patterns: a randomized controlled trial. Eur J Prev Cardiol 2017 Jan;9:69-78. [doi: 10.1016/j.eujim.2016.11.008]

38. Hwang R, Bruning J, Morris NR, Mandrusiak A, Russell T. Home-based telerehabilitation is not inferior to a centre-based program in patients with chronic heart failure: a randomised trial. J Physiother 2017 Apr;63(2):101-107 [FREE Full text] [doi: 10.1016/j.jphys.2017.02.017] [Medline: 28336297]

39. Fang J, Huang B, Xu D, Li J, Au WW. Innovative application of a home-based and remote sensing cardiac rehabilitation protocol in Chinese patients after percutaneous coronary intervention. Telemed J E Health 2019 Apr;25(4):288-293. [doi: 10.1089/tmj.2018.0064] [Medline: 30192210]

40. Harzand A, Witbrodt B, Davis-Watts ML, Alrohaibani A, Goese D, Wenger NK, et al. Feasibility of a smartphone-enabled cardiac rehabilitation program in male veterans with previous clinical evidence of coronary heart disease. Am J Cardiol 2018 Nov 01;122(9):1471-1476 [FREE Full text] [doi: 10.1016/j.amjcard.2018.07.028] [Medline: 30217377]

41. Maddison R, Rawstorn JC, Stewart RAH, Benatar J, Whittaker R, Rolleston A, et al. Effects and costs of real-time cardiac telerehabilitation: randomised controlled non-inferiority trial. Heart 2019 Jan;105(2):122-129 [FREE Full text] [doi: 10.1136/heartjnl-2018-313189] [Medline: $\underline{\text { 30150328] }}$

42. Peng X, Su Y, Hu Z, Sun X, Li X, Dolansky MA, et al. Home-based telehealth exercise training program in Chinese patients with heart failure: A randomized controlled trial. Medicine (Baltimore) 2018 Aug;97(35):e12069 [FREE Full text] [doi: 10.1097/MD.0000000000012069] [Medline: 30170422]

43. Rawstorn JC, Gant N, Rolleston A, Whittaker R, Stewart R, Benatar J, et al. End users want alternative intervention delivery models: usability and acceptability of the remote-cr exercise-based cardiac telerehabilitation program. Arch Phys Med Rehabil 2018 Nov;99(11):2373-2377. [doi: 10.1016/j.apmr.2018.06.027] [Medline: $\underline{30076800]}$

44. Anderson L, Sharp GA, Norton RJ, Dalal H, Dean SG, Jolly K, et al. Home-based versus centre-based cardiac rehabilitation. Cochrane Database Syst Rev 2017 Jun 30;6:CD007130 [FREE Full text] [doi: 10.1002/14651858.CD007130.pub4] [Medline: 28665511]

45. Huang K, Liu W, He D, Huang B, Xiao D, Peng Y, et al. Telehealth interventions versus center-based cardiac rehabilitation of coronary artery disease: a systematic review and meta-analysis. Eur J Prev Cardiol 2015 Aug;22(8):959-971. [doi: 10.1177/2047487314561168] [Medline: 25488550]

46. Rawstorn JC, Gant N, Direito A, Beckmann C, Maddison R. Telehealth exercise-based cardiac rehabilitation: a systematic review and meta-analysis. Heart 2016 Aug 01;102(15):1183-1192. [doi: 10.1136/heartjnl-2015-308966] [Medline: 26936337]

47. Su JJ, Yu DSF, Paguio JT. Effect of eHealth cardiac rehabilitation on health outcomes of coronary heart disease patients: a systematic review and meta-analysis. J Adv Nurs 2020 Mar;76(3):754-772. [doi: 10.1111/jan.14272] [Medline: 31769527$]$

48. Emerging technologies to support an aging population. TASK FORCE ON RESEARCH AND DEVELOPMENT FOR TECHNOLOGY TO SUPPORT AGING ADULTS 2019:1-40 [FREE Full text]

49. Andrews JA, Brown LJ, Hawley MS, Astell AJ. Older adults' perspectives on using digital technology to maintain good mental health: interactive group study. J Med Internet Res 2019 Feb 13;21(2):e11694 [FREE Full text] [doi: 10.2196/11694] [Medline: 30758292]

50. Fischer SH, David D, Crotty BH, Dierks M, Safran C. Acceptance and use of health information technology by community-dwelling elders. Int J Med Inform 2014 Sep;83(9):624-635 [FREE Full text] [doi: 10.1016/j.ijmedinf.2014.06.005] [Medline: 24996581]

51. Duscha BD, Piner LW, Patel MP, Craig KP, Brady M, McGarrah RW, et al. Effects of a 12-week mHealth program on peak VO and physical activity patterns after completing cardiac rehabilitation: a randomized controlled trial. Am Heart J 2018 May;199:105-114. [doi: 10.1016/j.ahj.2018.02.001] [Medline: 29754647]

52. Identifier: N. Longterm follow-up of cardiac patients with an smartphone-application -a randomized clinical study. - 2017:1.

53. Medical Research C. Creating an Optimized Technology-Based Weight Loss Program for Cardiac Rehabilitation Patients. CMR 2000 Jan 01:1. [doi: 10.31525/ct1-nct03845283]

54. Networks Inc. K. Rehabilitation at home using mobile health in older adults after hospitalization for Ischemic Heart Disease (resilient). Study Record Detail 2019 Jun 06:-. [doi: 10.31525/ct1-nct03978130] 
55. Salvi D, Ottaviano M, Muuraiskangas S, Martínez-Romero A, Vera-Muñoz C, Triantafyllidis A, et al. An m-Health system for education and motivation in cardiac rehabilitation: the experience of HeartCycle guided exercise. J Telemed Telecare 2018 May;24(4):303-316. [doi: 10.1177/1357633X17697501] [Medline: 28350282]

\author{
Abbreviations \\ AACVPR: American Association of Cardiovascular and Pulmonary Rehabilitation \\ ACC: American College of Cardiology \\ AHA: American Heart Association \\ CR: cardiac rehabilitation \\ CVD: cardiovascular disease \\ mHealth: mobile health \\ NHLBI: National Heart, Lung, and Blood Institute \\ NIH: National Institutes of Health \\ NINR: National Institute of Nursing Research \\ QoL: quality of life
}

Edited by G Eysenbach; submitted 17.03.20; peer-reviewed by L Quinlan, A Triantafyllidis, F Holl, M Mars, C Carrion; comments
to author 29.06.20; revised version received 22.11.20; accepted 07.12.20; published 08.02.21
Please cite as:
Wongvibulsin S, Habeos EE, Huynh PP, Xun H, Shan R, Porosnicu Rodriguez KA, Wang J, Gandapur YK, Osuji N, Shah LM, Spaulding
EM, Hung G, Knowles K, Yang WE, Marvel FA, Levin E, Maron DJ, Gordon NF, Martin SS
Digital Health Interventions for Cardiac Rehabilitation: Systematic Literature Review
J Med Internet Res 2021;23(2):e18773
URL: $\underline{\text { https://www.jmir.org/2021/2/e18773 }}$
doi: $10.2196 / 18773$
PMID: $\underline{3555259}$

(CShannon Wongvibulsin, Evagelia E Habeos, Pauline P Huynh, Helen Xun, Rongzi Shan, Kori A Porosnicu Rodriguez, Jane Wang, Yousuf K Gandapur, Ngozi Osuji, Lochan M Shah, Erin M Spaulding, George Hung, Kellen Knowles, William E Yang, Francoise A Marvel, Eleanor Levin, David J Maron, Neil F Gordon, Seth S Martin. Originally published in the Journal of Medical Internet Research (http://www.jmir.org), 08.02.2021. This is an open-access article distributed under the terms of the Creative Commons Attribution License (https://creativecommons.org/licenses/by/4.0/), which permits unrestricted use, distribution, and reproduction in any medium, provided the original work, first published in the Journal of Medical Internet Research, is properly cited. The complete bibliographic information, a link to the original publication on http://www.jmir.org/, as well as this copyright and license information must be included. 